\title{
Generalised single particle models for high-rate operation of graded lithium-ion electrodes: systematic derivation and validation
}

\author{
G. Richardson ${ }^{1,2,5}$, I. Korotkin ${ }^{1,2,6}$, R. Ranom ${ }^{3}$, M. Castle ${ }^{4}$, and J. M. \\ Foster $^{2,4,7}$
}

${ }^{1}$ Mathematical Sciences, University of Southampton, University Rd., SO17 1BJ, UK

${ }^{2}$ The Faraday Institution, Quad One, Becquerel Avenue, Harwell Campus, Didcot, OX11 0RA, UK

${ }^{3}$ Faculty of Electrical Engineering, Universiti Teknikal Malaysia Melaka, 76100 Melaka, Malaysia

${ }^{4}$ School and Mathematics and Physics, University of Portsmouth, Lion

Terrace, PO1 3HF, UK

${ }^{5}$ g.richardson@soton.ac.uk

${ }^{6}$ i.korotkin@soton.ac.uk

${ }^{7}$ jamie.michael.foster@gmail.com

February 12, 2020

\begin{abstract}
A derivation of the single particle (SP) model is made from a Doyle-Fuller-Newman (DFN) model for electrodes composed of uniformly sized spherical electrode particles of one chemistry. The derivation uses a formal asymptotic method based on the disparity between the size of the thermal voltage and that of the characteristic change in overpotential that occurs during (de)lithiation. Comparison is made between the SP model and the DFN model for electrodes made from: lithium nickel manganese cobalt oxide (NMC), graphite and lithium iron phosphate (LFP). These are used to identify regimes where the SP model is accurate. For most chemistries, even at moderate rates, there are discrepancies between the DFN model and the SP model due to spatial non-uniformities in the electrolyte. Motivated by these discrepancies a correction term to the SP model is derived. Incorporating this into the SP model gives the corrected SP (cSP) model whose accuracy is very significantly improved over the SP model. Generalised versions of the cSP model for graded electrodes containing multiple electrode particle sizes (or chemistries) in different regions of the electrode, are also
\end{abstract}


derived. The results of this generalisation to the cSP model compare favourably to the full DFN model, even at relatively high discharge rates, where the active electrode material is either graphite or a particular NMC variant.

\section{Introduction}

Lithium-ion batteries (LIBs) provide rechargeable energy storage at an unrivalled energy and power density, with a high cell voltage, and a slow loss of charge when not in use [5]. These characteristics mean that they are already ubiquitous in the consumer electronics sector and are being increasingly adopted for use in electric vehicles and off grid storage. However, particularly for vehicular propulsion, there are still major hurdles to be overcome in terms of lengthening service life, facilitating higher (dis)charge rates, and improving safety, particularly in high-rate regimes $[44,45]$. Driven largely by the incumbent legislation to ban the combustion engine across large parts of the world before 2040, it has been predicted that the demand for LIBs will balloon from 45GWh/year (in 2015) to one of 390GWh/year in 2030 [50] and so improvements in LIB performance are needed as a matter of urgency.

A LIB pack is comprised of a collection of electrochemical cells each of which is comprised of a positive and a negative porous electrode separated by an inert porous spacer (the separator). In many cases this assembly is bathed in a liquid electrolyte, and this is the cell design we consider here, but other architectures exists where a solid electrolyte is used [49]. Both electrodes are composites, comprised of a porous network of microscopic electrode particles (into which $\mathrm{Li}^{+}$can intercalate), a nanoparticulate conductive additive and an inert polymer binder. The voids within porous electrode scaffolds are filled with liquid electrolyte. During discharge, $\mathrm{Li}^{+}$ions deintercalate from the negative electrode material, flow through the electrolyte, across the separator, and intercalate into the positive electrode material on the other side of the cell. This ionic current across the cell is compensated by a flow of free electrons through the external circuit which provides useful electric current. The charging process occurs similarly but with the ionic and electronic currents flows being reversed.

Aspects of LIB performance are modelled over a wide range of length scales ranging from atomistic simulations of battery materials to full device simulations (see [18] for details). The current work focusses on electrode scale models of LIBs and is based on the Doyle-FullerNewman approach to modelling an individual cell; this was developed by Newman and his co-workers in the mid 90's and early 2000's [12, 13, 19, 39, 43]. It has since been formally justified using asymptotic homogenisation techniques [37, 40]. In this approach Lithium ion transport is considered on two scales, a macroscopic scale for ion transport across the cell through the electrolyte, and a microscopic scale, for transport of ions within individual electrode particles. The coupling between the two scales takes place via a reaction term on the surface of the electrode particles that describes the intercalation and de-intercalation of $\mathrm{Li}^{+}$ions taking place there. In the original Doyle-Fuller-Newman (DFN) works the electrode is assumed to have a one-dimensional slab-like geometry and the electrode particles are all assumed to be spherically symmetric so that both the macro- and microscopic problems are effectively one-dimensional in space, and the resulting coupled problem is pseudo-twodimensional [22].

The original DFN model uses a simple linear diffusion equation to model lithium transport 
within the electrode particles. With improvements in the understanding of the chemistry of these active (electrode) materials it has become clear that linear diffusion is not a good description of $\mathrm{Li}^{+}$ion transport within these materials [20]. Recent works have focussed on incorporating more realistic lithium transport models in the active materials into DFN. These range from nonlinear diffusion models calibrated against experimental data [14, 15, 23] to Cahn-Hilliard models for phase change materials $[9,11,16]$. Here we employ the former approach and restrict our attention to nonlinear diffusion models for $\mathrm{Li}^{+}$ion transport in the active materials. The original DFN formulation has also been generalised to higher dimensions. Thus, for example, electrode particle shape effects can be investigated with a 2or 3-dimensional microscale problem, while spatially inhomogeneities in electrode discharge, arising from non-uniform heating or the positioning of the electrode tabs [17, 24], can be studied using a 2- or 3-dimensional macroscale problem.

Whilst it is difficult to overstate the success and utility of the DFN approach, it can be criticised for being relatively expensive to solve. This is a particularly significant difficulty when it is being used as a tool to optimise cell design [7], to model 2- or 3-macroscopic dimensions [10, 47], as a tool in parameter estimation studies [4, 41], or to implement realtime accurate models on the relatively modest computing resources found in electric vehicles. Its multiscale nature means that the underlying equations are posed over two separate spatial dimensions and, since these equations are nonlinear, obtaining solutions is a task that needs to be tackled numerically. This has motivated many authors to consider simplified versions of the DFN. Perhaps the most well-known model of this type is the single particle model, or SP model, which results from assuming that the electrode particles are uniformly sized spheres, and then arguing that the electrochemical reactions occur roughly uniformly, throughout each electrode, so that the active material in any particle (in a given electrode) (de)lithitates at a rate that is independent of the position of the particle in the electrode [27, 35]. In this way, finding model solutions is reduced to the task of solving a single spherical transport problem inside a 'representative' particle in each electrode. In this context we note that the thesis of Ranom [36], from which the current work stems, contains a systematic derivation of the SP model from the DFN model.

After completion of this work, see [38], we became aware of another article in-progress that employs asymptotic methods to simplify the DFN model [26]. In [26], the asymptotic limit of large electrode and electrolyte conductivities and large electrolyte diffusivity is taken; this is a different limit to the one taken here. Their limit recovers the SP model at leading order because the gradients in the electrolyte concentration, electrolyte potential, and electrode potentials are all small and this gives rise to homogeneous behaviour of the electrode particles. Contrastingly, in our limit, the variation in the overpotential across the electrode is small in comparison the typical variation in the equilibrium potential as lithium is removed/inserted into the electrode and it is this that leads to the SP model. Whilst both limits recover an SP model at leading order, the correction terms are different. We note that with the possible exception of LFP our approach is considerably more accurate than that of [26] and is able to accurately capture discharges of between $12 \mathrm{C}$ and $16 \mathrm{C}$ for graphite and NMC when validated against solutions to the DFN model; this compares to the corrected single particle model in [26] which begins to show significant divergence from solution to the DFN model for their trial cell at discharge rates of around $3 \mathrm{C}$ and above. Furthermore, in [26], the corrected single particle model is only compared to an unrealistic version of the DFN model in which lithium 
transport in the electrode material is assumed linear whereas the corrected single particle model derived here is compared to realistic DFN models in which the diffusivity of lithium in the electrode materials has been calibrated against real data, obtained from [14, 15], and is highly nonlinear. In order to better illustrate the relative accuracy of these different single particle approaches we make a comparison between the results for a galvanostatic discharge of a full cell obtained from our corrected SP model, those from the corrected SP model of [26], and those from the DFN model; the results are displayed in Figure 9. In all three cases we use the same realistic data for the physical properties of the cells (taken from the work of [15]). We are able to show that the agreement between the DFN model results and those from our corrected SP model is much better than that between the DFN model and the corrected $\mathrm{SP}$ model derived in [26]. Indeed our corrected SP model is almost indistinguishable from the DFN model up to a discharge rate of $7.5 \mathrm{C}$. A further advantage of our approach, over that of [26], is that it can be accurately extended to spatially non-uniform electrodes with multiple particle sizes and or chemistries.

We also note the work of Moyles et al. [28] which derives an asymptotic reduction of a DFN model, but does so by first volume averaging lithium transport over the electrode particles in the DFN model, and thereby implicitly assumes transport within individual electrode particles is rapid.

In order to investigate the validity of the corrected SP model approximation for different electrode chemistries we will, throughout the main body of this work, restrict attention to a half-cell configuration. The extension to the full cell configuration is relatively straightforward, and is given in appendix B. The half-cell configuration is comprised of a single porous electrode (either a cathode or anode) being (dis)charged, through a separator, against a metallic Li counter electrode [32, 46], see figure 1. In order to derive a generalised version of the SP model, applicable to graded electrodes, we allow the electrode to contain a spatial distribution of electrode particle sizes and/or chemistries (as illustrated in Figure 1). Motivated by the need to provide a useful tool for the practitioners, we validate our approximations, as far as possible, against realistic data sets for the DFN model. Here these data sets are primarily based upon two works from the Ecker group [14, 15] which adopt a combined theoretical and experimental approach in which they validate their DFN simulations against data collected from real cells. Validation of the full cell cSP model against the full cell DFN model and against Ecker's experimental data is carried out in appendix B.

In the next section of the paper we describe the DFN model as well as the boundary and initial conditions with which it should be supplemented in order to mimic a half-cell configuration. Then, in $\S 3$ we describe the multiparticle (MP) model, which is a generalisation of the standard SP model that allows for graded electrodes and ones with multiple electrode particle chemistries. We also outline how higher order terms, which capture the effects of spatial and temporal variations in the electrolyte properties, can be incorporated into the MP model. The inclusion of these higher order terms requires that a one-dimensional system of PDEs be solved in the electrolyte, in addition to the one-dimensional nonlinear diffusion equations for $\mathrm{Li}^{+}$ion transport in the representative particles in each electrode. However the extra complexity arising from the introduction of the electrolyte equation into the generalised SP model is outweighed by the significant enhancement in accuracy that this allows us to obtain. This is demonstrated with the aid of some examples for: (i) electrodes with a uniform particle size and (ii) graded electrodes with two sizes of particle segregated into 
separate regions of the electrode (an electrode architecture that is suggested as a means to enhance performance [29]). In $\S 4$ we introduce scalings and rewrite the DFN in dimensionless form. This nondimensionalisation facilitates the asymptotic analysis which is the subject of $\S 5$. Here, the SP and MP models, and their higher order correction terms, are derived systematically from the DFN. The generalisation of the SP and MP models to a full cell are presented in appendix B. Finally, in $\S 6$ we draw our conclusions.

\section{Problem formulation}

Here, we consider a DFN model posed on the half-cell model geometry shown in figure 1. It is assumed that all electrode particles are spherical. To allow for the possibility that the electrode is graded the particle radii, $r=R$, are allowed to vary across the electrode (i.e. $R=R(x)$ ). We do not include capacitive effects in the model and instead focus on dynamics occurring on the timescale of the cell (dis)charge, which is much longer than the capacitive timescale. For more details on modelling capacitive effects see $[2,3,21]$.

Electrolyte equations. The equations governing ionic transport through the electrolyte are discussed in detail in [30] and are based on the Stefan-Maxwell-Onsager equations. Here we restrict our attention to a 1:1 electrolyte (concentration $c$ ) comprised of a solvent, lithium ions and a generic negative counterion. The relevant ion transport equation through the electrolyte-filled pore space is

$$
\epsilon_{l}(x) \frac{\partial c}{\partial t}+\frac{\partial N_{-}}{\partial x}=0, \quad N_{-}=-\mathcal{B}(x) D(c) \frac{\partial c}{\partial x}-\left(1-t^{+}\right) \frac{j}{F} \quad \text { in } \quad-L_{s}<x<L .
$$

where, $x$ and $t$ denote position through the electrode and time respectively, $\epsilon_{l}$ is the local volume fraction of electrolyte, $N_{-}$is the effective flux of negative counterions across the electrolyte, $D$ is the ionic diffusivity of the electrolyte, $\mathcal{B}$ is the permeability factor (often estimated using the ad-hoc relation offered by Bruggeman which takes $\left.\mathcal{B}=\epsilon_{l}^{1.5}[6]\right), t^{+}$is the transference number, $j$ is the ionic current density and $F$ is Faraday's constant. In contrast to other works, the ion transport equation is formulated in terms of the anion flux, $N_{-}$, rather than the flux of lithium ions, $N_{+}$. This gives rise to an equation in conservation form, without the source/sink terms that are required in the alternative form to account for the (de)intercalation of lithium ions. As a consequence it is relatively straightforward to obtain numerical solutions to (1) that accurately conserve ions within the electrolyte. The ionic current density $j$ satisfies a current conservation law, with a source to describe current transfer from the electrode, and the electrolytic analogue of Ohm's law

$$
\frac{\partial j}{\partial x}=b(x) j_{n}, \quad j=-\mathcal{B}(x) \kappa(c)\left(\frac{\partial \Phi}{\partial x}-\frac{2 R_{g} T}{F} \frac{1-t^{+}}{c} \frac{\partial c}{\partial x}\right) \quad \text { in } \quad-L_{s}<x<L .
$$

Here $b$ is the Brunauer-Emmett-Teller (BET) surface area (with units of $1 / m$ ), $\kappa$ is the ionic conductivity, $\Phi$ is the electric potential in the electrolyte, $R_{g}$ the (molar/universal/ideal) gas constant, $T$ absolute temperature and $j_{n}$ is the reaction current density (i.e. the current density flowing though the surface of the electrode particles into the electrolyte). The latter is zero in the separator and given by the Butler-Volmer equations in the electrode. Charge 
transport through the (solid) matrix of electrode particles, binder and conducting additives is described by Ohms law with a sink term to describe the transfer of current into the electrolyte

$$
\frac{\partial j_{s}}{\partial x}=-b(x) j_{n}, \quad j_{s}=-\sigma \frac{\partial \Phi_{s}}{\partial x} \quad \text { in } \quad 0<x<L
$$

Here $\Phi_{s}, j_{s}$ and $\sigma$ are the electric potential, current density and conductivity of the electrode. Equations (1)-(3) depend only on the macroscopic spatial variable, $x$, measuring distance across the electrode, and are hence referred to as the macroscopic equations (cf. figure 1).

Boundary conditions It is assumed that the half-cell (dis)charges according to some specified current supply/demand, $I(t)$, and as such appropriate boundary conditions on the sixth order system of PDEs posed on the macroscale, (1)-(3), are that: (i) the Li-metal counter-electrode supplies an ionic current density of $I(t) / A$ to the electrolyte on $x=-L_{s}$; (ii) current of density $I(t) / A$ is extracted from the solid phase of the electrode at the current collector $x=L$; and (iii) on $x=0$ no current flows from the solid phase into the separator. In addition (iv) the potential $\Phi$ in the electrolyte is specified on $x=-L_{s}$ (edge of the separator) and (v) and (vi) there is no counter ion flux across the interfaces between the electrolyte and the separator and between the electrolyte and the current collector. In summary this gives

$$
\begin{array}{r}
\left.j\right|_{x=-L_{s}}=\frac{I(t)}{A},\left.\quad N_{-}\right|_{x=-L_{s}}=0,\left.\quad \Phi\right|_{x=-L_{s}}=0 \\
\left.j_{s}\right|_{x=0}=0,\left.\quad j_{s}\right|_{x=L}=\frac{I(t)}{A},\left.\quad N_{-}\right|_{x=L}=0 .
\end{array}
$$

The Butler-Volmer reaction rate It remains to specify the reaction current density $j_{n}$. In the separator $\left(-L_{s}<x<0\right)$, where there are no electrode particles, this is zero. In the electrode $(0 \leq x<L)$ the reaction rate is determined by the Butler-Volmer (BV) equation $[19,30,31]$ such that $j_{n}$ is given by

$$
j_{n}=\left\{\begin{array}{cc}
0 & \text { in }-L_{s}<x<0, \\
2 F k c^{1 / 2}\left(\left.c_{s}\right|_{r=R(x)}\right)^{1 / 2}\left(c_{s}^{\max }-\left.c_{s}\right|_{r=R(x)}\right)^{1 / 2} \sinh \left(\frac{F \eta}{2 R_{g} T}\right) & \text { in } \quad 0 \leq x<L,
\end{array}\right.
$$

where the overpotential is given by

$$
\eta=\Phi_{s}-\Phi-U_{\mathrm{eq}}\left(\left.c_{s}\right|_{r=R(x)}\right) .
$$

Here, $U_{\text {eq }}, c_{s}^{\max }$ and $R(x)$ are the equilibrium potential, the maximum concentration of lithium that can be stored in the electrode material, and the radii of electrode particles.

Equations on the microstructure Evaluating the reaction current density, $j_{n}$, necessitates solving for the solid-state lithium concentration on the surfaces of the electrode particles throughout the electrode. Whilst there is considerable debate about the correct solid state transport model, particularly for phases separating electrode materials [1, 25, 33], here, we opt to model solid state lithium transport as a non-linear diffusion process. Thus, where the 


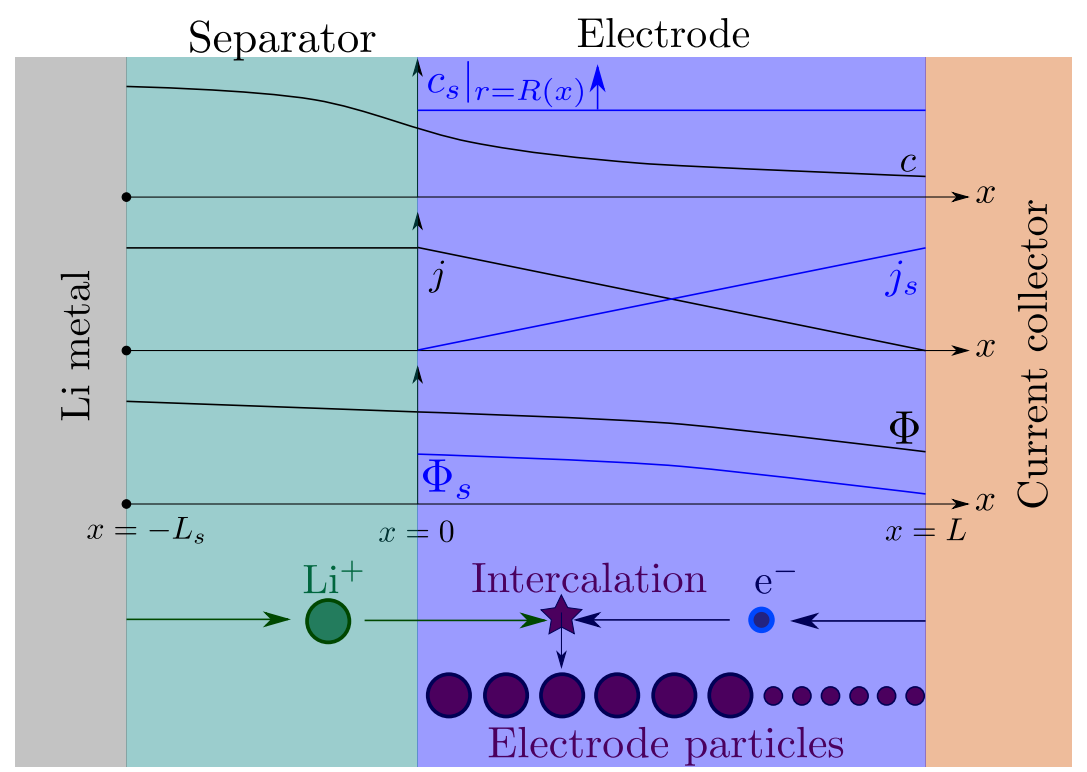

Figure 1: A schematic of the half cell geometry as well as the independent variables and snapshot of their qualitative profiles during charging if the electrode is an anode, or discharging if the electrode is a cathode. Here, $c$ and $c_{s}$ denote the concentrations of $\mathrm{Li}^{+}$ion in the electrolyte and $\mathrm{Li}$ in the electrode material, $j$ and $j_{s}$ are the current densities in the electrolyte and solid respectively, and $\Phi$ and $\Phi_{s}$ are the potentials in the electrolyte and solid respectively.

electrode particles are spherical with a graded distribution of particle radii $r=R(x)$, solid state lithium transport is modelled by

$$
\frac{\partial c_{s}}{\partial t}=\frac{1}{r^{2}} \frac{\partial}{\partial r}\left(r^{2} D_{s}\left(c_{s}\right) \frac{\partial c_{s}}{\partial r}\right) \quad \text { in } \quad 0<r<R(x) \text { and } 0<x<L
$$

were $D_{s}$ is the concentration-dependent diffusivity and $r$ measures distance from the centre of a particle. Equations (8) are closed by the boundary conditions

$$
c_{s} \text { bounded on } r=0, \quad-\left.D_{s}\left(c_{s}\right) \frac{\partial c_{s}}{\partial r}\right|_{r=R(x)}=\frac{j_{n}}{F} .
$$

The former is a regularity conditions required to eliminate singular behaviour at the origin whilst the latter relates lithium ion flux across the particle surface to the reaction current density $j_{n}$. Equations (8)-(9) are referred to as the microscopic transport problem.

The half-cell potential The half-cell potential, $V$, is given by the expression

$$
V(t)=\left.\Phi_{s}\right|_{x=L}
$$

It is straightforward to include an Ohmic drop across the current collector interface, caused by a contact resistance $\mathcal{R}$, by replacing this expression by one of the form $V(t)=\left.\Phi_{s}\right|_{x=L}-\mathcal{R} I$. 
Initial conditions The parabolic equations (1) and (8) require initial conditions on $c$ and on $c_{s}$. Assuming that the electrode is initially in equilibrium, so that both $I(0)=0$ and $j_{n}(x, 0)=0$, leads to the requirement of a uniform electrolyte salt concentration

$$
\left.c\right|_{t=0}=c_{\text {init }} \quad \text { in } \quad-L_{s}<x<L .
$$

and a uniform lithium concentration in all the electrode particles

$$
\left.c_{s}\right|_{t=0}=c_{s, \text { init }} \quad \text { in } 0<r<R(x) \text { and } 0<x<L .
$$

These conditions, together with the model, imply that $\Phi(x, 0)=0$ and $\Phi_{s}(x, 0)=U_{\text {eq }}\left(c_{s, \text { init }}\right)$.

Relations between microstructural parameters. Under the assumptions that the electrode particles are spherical, and that the entirety of their internal volume and surface area are available for intercalation the electrode particle radius $R(x)$, BET surface area $b(x)$, and electrode particle volume fraction $\epsilon_{s}$ are related to each other by the expression

$$
\epsilon_{s}(x)=\frac{b(x) R(x)}{3} .
$$

This relationship follows from noting that the volume fraction of particles within a small representative elementary volume is the product of the volume of a single particle, namely $4 \pi R^{3} / 3$, and the number of particles within the REV, namely $b /\left(4 \pi R^{2}\right)$. Furthermore the electrolyte volume fraction, $\epsilon_{l}$, is related to $\epsilon_{s}$ via

$$
\epsilon_{l}(x)=1-\epsilon_{\text {inert }}(x)-\epsilon_{s}(x),
$$

where $\epsilon_{\text {inert }}$ is the volume fraction of electrochemically inert material (e.g., the polymer binder or the conductivity enhancing additive).

\section{Results}

In this section results of our analysis of the DFN model (1)-(14) are stated and comparison is made between the simplified single particle (SP) models that we derive and numerical solutions to the DFN model. Formal justification of the simplified SP models is left until §4-5. We begin by stating the generalisation of the SP model approximation to graded electrodes, we term this the multi-particle (MP) model. This is based on the assumption that $\mathcal{U}$ the characteristic change in overpotential that occurs as lithium is intercalated into (or removed from) the electrode material is much larger than the thermal voltage $V_{T}=R_{g} T / F \approx 26 \mathrm{mV}$. Formally we require

$$
\lambda \ll 1 \quad \text { where } \quad \lambda=\frac{F \mathcal{U}}{R_{g} T} .
$$

It is thus applicable to materials such as graphite, NMC or LCO (though not to LFP where $\mathcal{U} \approx 26 \mathrm{mV})$. We then give a recipe for extending the SP and MP models to incorporate further terms, which depend upon the electrolyte behaviour, and considerably enhance the 

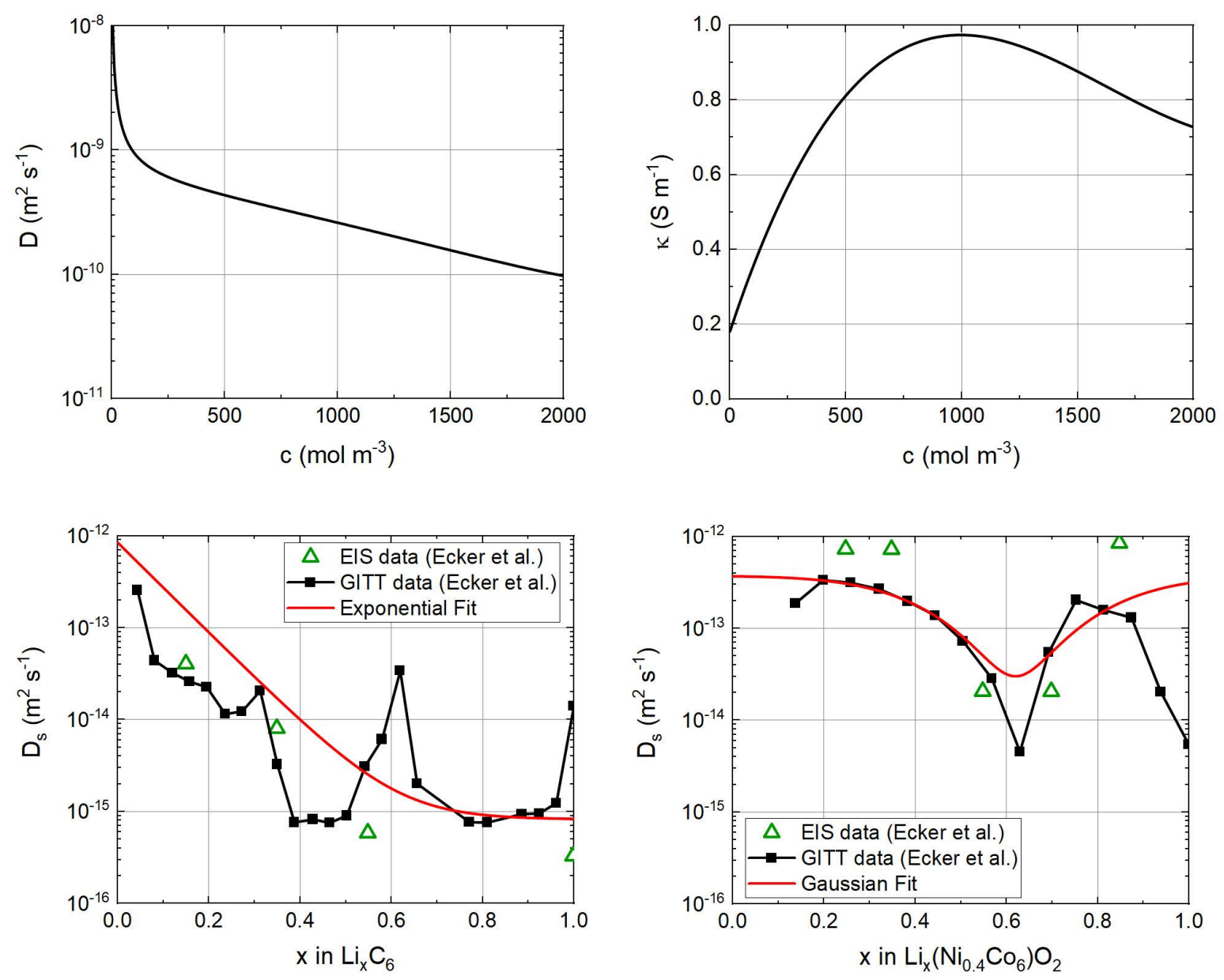

Figure 2: Ionic diffusivity (top left) and conductivity (top right) of the electrolyte from [14], non-linear diffusivity of graphite $\left(\mathrm{LiC}_{6}\right)$ anode (bottom left) and $\mathrm{Li}\left(\mathrm{Ni}_{0.4} \mathrm{Co}_{0.6}\right) \mathrm{O}_{2}$ cathode (bottom right). Experimental data from [14] and approximate fits.

accuracy of the method. Henceforth we will refer to the corrected SP and MP models, which include the additional terms, as the 'corrected SP (cSP) corrected MP (cMP) model'. In order to illustrate the method we consider two examples. The first of these (described in §3.1) is the SP model which applies to electrodes comprised of uniform sized electrode particles (i.e. with no grading) while the second (described in §3.2) is the MP model which applies to electrodes which are graded with different particle sizes occupying different regions of the electrode (we consider a case in which there is one size of particle near the separator and one near the current collector). In both cases we compare results of the SP/MP model and cSP/cMP model to the full numerical solution to the DFN model (1)-(10) for electrodes of several different chemistries.

The leading order approximation. The most basic approximation to the Doyle-FullerNewman model (1)-(10) in the scenario described above is the following multi-particle (MP) 

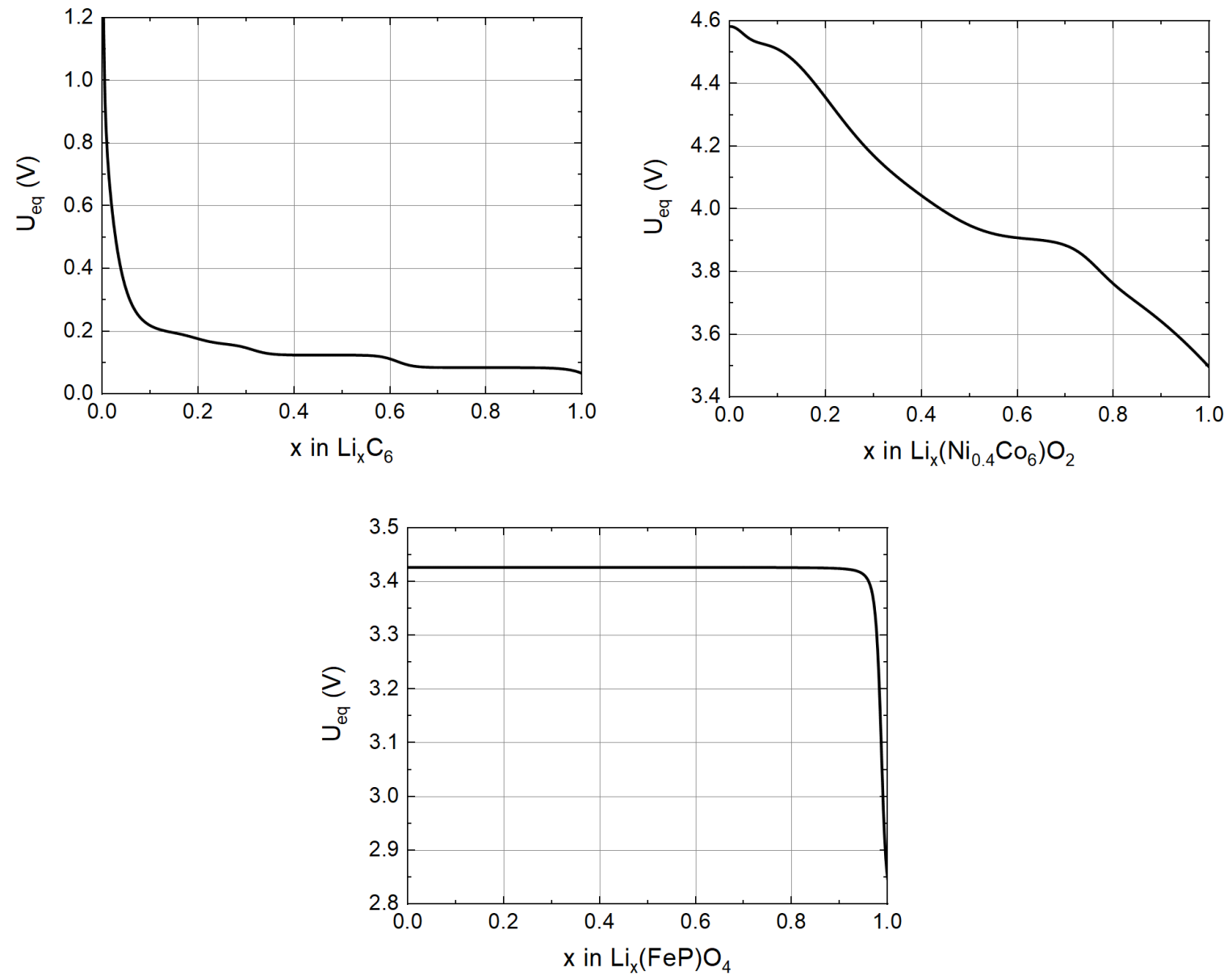

Figure 3: Equilibrium potential of graphite $\left(\mathrm{LiC}_{6}\right)$ anode [14] (top left), $\mathrm{Li}\left(\mathrm{Ni}_{0.4} \mathrm{Co}_{0.6}\right) \mathrm{O}_{2}$ cathode [14] (top right), and LFP $\left(\mathrm{LiFePO}_{4}\right)$ electrode [36] (bottom). 
generalisation to the SP model:

$$
\begin{gathered}
\frac{\partial c_{s}}{\partial t}=\frac{1}{r^{2}} \frac{\partial}{\partial r}\left(r^{2} D_{s}\left(c_{s}\right) \frac{\partial c_{s}}{\partial r}\right) \quad \text { in } \quad 0 \leq r \leq R(x), \\
c_{s} \text { bounded on } r=0,\left.\quad c_{s}\right|_{r=R(x)}=\mathcal{C}(t),\left.\quad c_{s}\right|_{t=0}=c_{s, \text { init }}, \\
\int_{0}^{L} b(x) j_{n}(x, t) d x=-\frac{I(t)}{A} \quad \text { where } \quad j_{n}=-\left.F D_{s}\left(c_{s}\right) \frac{\partial c_{s}}{\partial r}\right|_{r=R(x)}
\end{gathered}
$$

Here the function $\mathcal{C}(t)$, which specifies the lithium concentration on the surface of the electrode particles, is chosen so that the integral constraint (18), which ensures that the total reaction is equal to the net current $I(t)$, is satisfied. The leading order approximation to the voltage, $V(t)$, of the half-cell is then calculated from the solution of this problem (16)-(18) via the relation

$$
V(t) \approx U_{\mathrm{eq}}(\mathcal{C}(t)),
$$

which is a leading order approximation to the Butler-Volmer equation (6). The key requirement for the MP model to hold, is that lithium concentration $\mathcal{C}(t)$ on the surface of all electrode particles is approximately equal (at any given time). This is a consequence of condition (15); if any particle has a surface lithium concentration that is significantly different to the that of the others, its overpotential also significantly differs from these particles. In turn this leads to an exponentially large Butler-Volmer reaction current $j_{n}$ on its surface (see (6)) which rapidly drives it back to the same surface concentration as the other particles. However, as we shall demonstrate, (19), the approximation to the cell voltage, is not particularly accurate at moderate to large discharge rates. This is mainly because the effects of the electrolyte on the Butler-Volmer reaction rate have been neglected. In order to incorporate these neglected effects a higher order approximation to the DFN model, derived in the subsequent section, is required.

A higher order approximation. A more accurate expression for $V(t)$ can be calculated from the solution to (16)-(18) but additionally requires a one-dimensional problem for the electrolyte be solved. This expression reads

$$
V(t) \approx U_{\mathrm{eq}}(\mathcal{C}(t))+\frac{\int_{0}^{L} b(x) R(x)\left[\eta(x, t)+\Phi(x, t)-\int_{x}^{L} \frac{j_{s}\left(x^{\prime}, t\right)}{\sigma\left(x^{\prime}\right)} d x^{\prime}\right] d x}{\int_{0}^{L} b(x) R(x) d x} .
$$

Here the potential in the electrolyte $\Phi(x, t)$, the associated overpotential $\eta(x, t)$, and $j_{s}(x, t)$ are calculated from the solution to the one dimensional electrolyte problem

$$
\begin{gathered}
\epsilon_{l}(x) \frac{\partial c}{\partial t}+\frac{\partial N_{-}}{\partial x}=0, \quad N_{-}=-\mathcal{B}(x) D(c) \frac{\partial c}{\partial x}-\left(1-t^{+}\right) \frac{j}{F}, \\
\frac{\partial j}{\partial x}=b(x) j_{n}(x, t), \quad j=-\mathcal{B}(x) \kappa(c)\left(\frac{\partial \Phi}{\partial x}-\frac{2 R_{g} T}{F} \frac{1-t^{+}}{c} \frac{\partial c}{\partial x}\right),
\end{gathered}
$$


with

$$
\begin{array}{r}
\left.j\right|_{x=-L_{s}}=\frac{I(t)}{A},\left.\quad N_{-}\right|_{x=-L_{s}}=0,\left.\quad \Phi\right|_{x=-L_{s}}=0,\left.\quad N_{-}\right|_{x=L}=0 \\
\text { and }\left.\quad c\right|_{t=0}=c_{\text {init }}
\end{array}
$$

where

$$
j_{s}(x, t)=\frac{I}{A}-j(x, t), \quad \eta(x, t)=2 \frac{R_{g} T}{F} \operatorname{arcsinh}\left(\frac{j_{n}(x, t)}{2 F k\left[\left(c_{s}^{\max }-\mathcal{C}(t)\right) \mathcal{C}(t) c\right]^{1 / 2}}\right),
$$

and $j_{n}(x, t)$ is obtained from the solution to the leading order problem (16)-(18). Notably the approximated expression for $V(t)$ calculated in $(20)$ is a formally accurate approximation to the DFN model (1)-(10) (as explained in $\S 5$ ), for all discharge rates, in the case that the electrode is composed of uniformly sized particles of a single material. However, even where this is not the case (e.g. if the electrode is graded), it is still formally accurate provided that the (dis)charge rate is not excessively large in comparison to the characteristic timescale for transport within the electrode particles. More specifically it is formally accurate if

$$
\mathcal{Q} \ll 1 \quad \text { where } \quad 3 \mathcal{Q}=\frac{\text { timescale for Li diffusion into electrode particle }}{\text { timescale for cell discharge }} .
$$

The dimensionless parameter $\mathcal{Q}$ will be defined rigorously below in $\S 4$. Since this requirement is usually satisfied, even at moderate to aggressive discharge rates, it typically offers a significant improvement over the leading order approximation (19).

\subsection{The Single Particle Model and comparison to the DFN model}

In the case where the electrode particles have uniform size $R$ the leading order equations (16)(18) simplify significantly, since $c_{s}=c_{s}(r, t)$ and $j_{n}=j_{n}(t)$. Thus only a single microscopic transport problem in $r$, in a representative particle, needs to be solved. This problem is comprised of (16) subject to the boundary conditions

$$
c_{s} \text { bounded on } r=0,\left.\quad D_{s}\left(c_{s}\right) \frac{\partial c_{s}}{\partial r}\right|_{r=R}=-\frac{j_{n}(t)}{F} \quad \text { where } \quad j_{n}(t)=\frac{I(t)}{A \int_{0}^{L} b(x) d x} \text {. }
$$

From the solution to this problem we can evaluate

$$
\mathcal{C}(t)=\left.c_{s}\right|_{r=R}
$$

and use the functions $j_{n}(t)$ and $\mathcal{C}(t)$, thus determined, as inputs in the electrolyte model (21)-(25). In turn this problem can be solved to obtain the data necessary to compute the corrected voltage via (20).

Simulations of both the basic SP model and its more accurate extension, the cSP model, are compared to simulations of the full DFN model in figure 4 as discharge curves showing cell voltage $V(t)$ plotted against time $t$ in the left-hand panels and $V(t)$ plotted against capacity in the right-hand panel. We examine three different electrode chemistries, namely, 

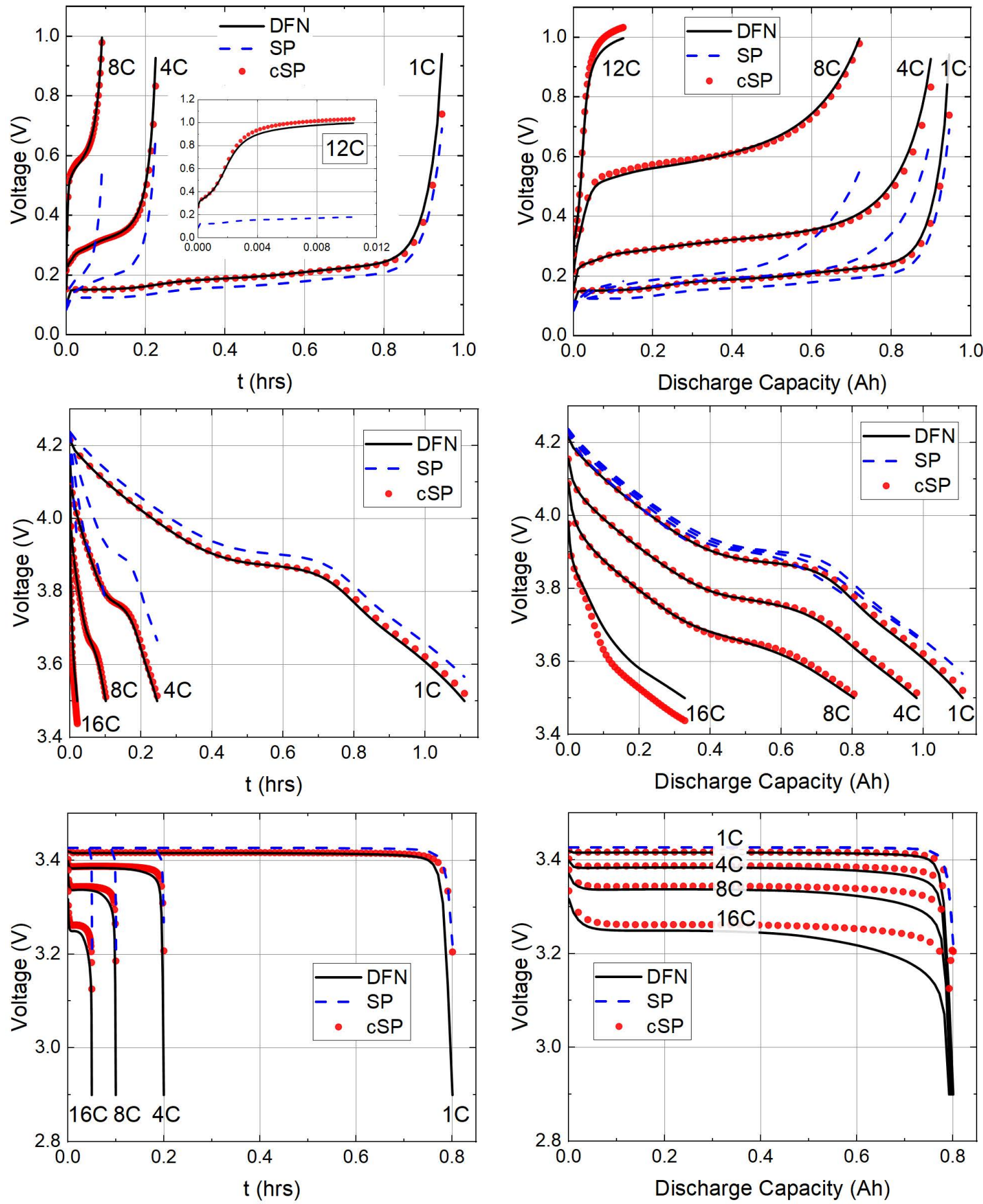

Figure 4: Cell voltages $V$ calculated using the full DFN model, SP model and cSP model for graphite $\left(\mathrm{LiC}_{6}\right)$ anode (top row), $\mathrm{Li}\left(\mathrm{Ni}_{0.4} \mathrm{Co}_{0.6}\right) \mathrm{O}_{2}$ cathode (middle row) and $\mathrm{LFP}\left(\mathrm{LiFePO}{ }_{4}\right)$ electrode (bottom row) at different discharge rates. Results are shown against both time (left) and discharge capacity (right). 

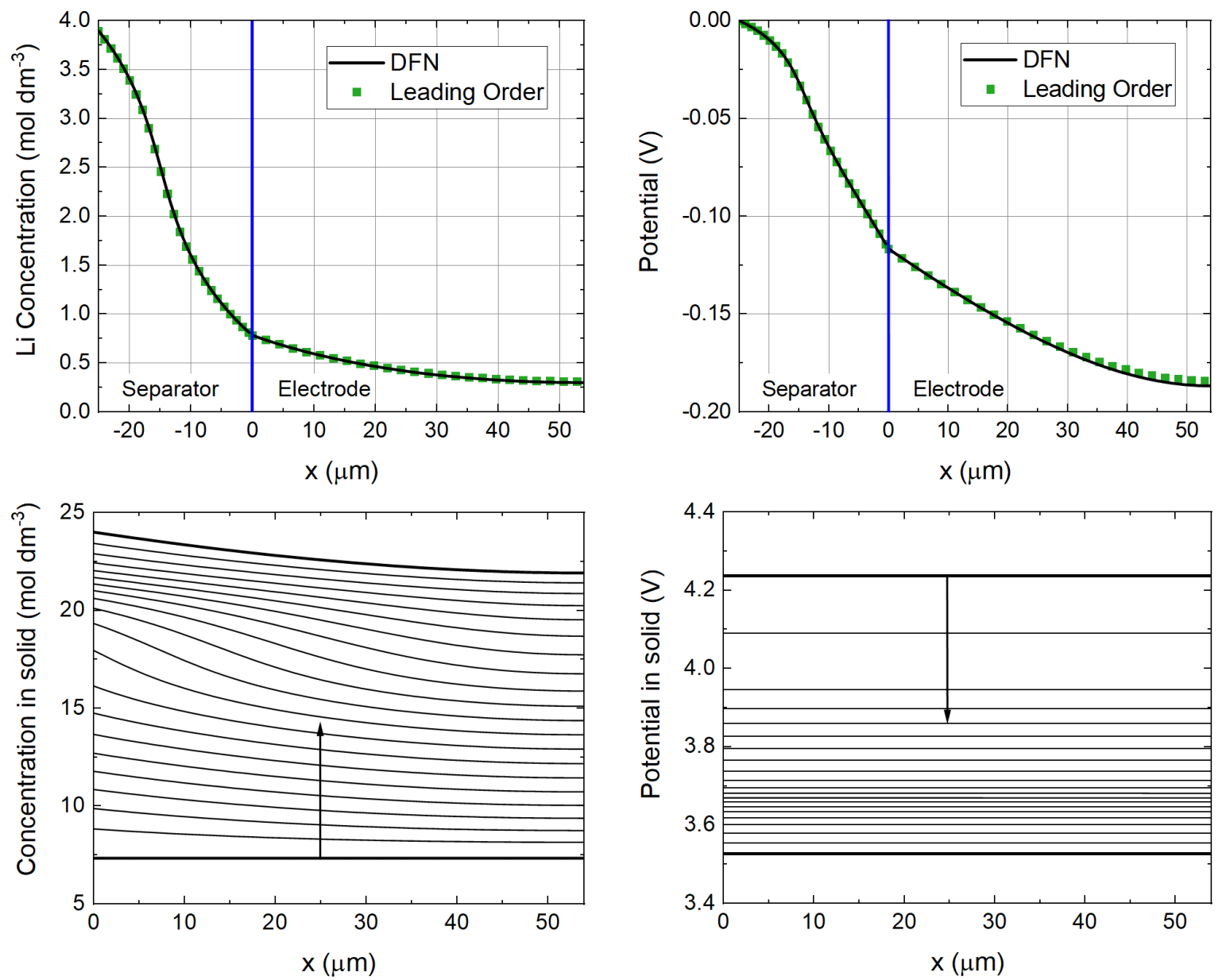

Figure 5: Concentration of $\mathrm{Li}^{+}$ions (top left) and potential (top right) in the electrolyte across a half-cell with a $\mathrm{Li}\left(\mathrm{Ni}_{0.4} \mathrm{Co}_{0.6}\right) \mathrm{O}_{2}$ electrode calculated using the DFN model and the leading order electrolyte equations, (21)-(25), at 8C discharge rate. In the upper panels a single snapshot in time (at the end of discharge) is shown because the electrolyte approaches a steady-state rapidly and hence the profiles at earlier times look very similar to those shown. Concentration of Lithium on the electrode particle surfaces (bottom left) and the potential in the solid (bottom right) across the $\mathrm{Li}\left(\mathrm{Ni}_{0.4} \mathrm{Co}_{0.6}\right) \mathrm{O}_{2}$ electrode calculated using the full DFN at $8 \mathrm{C}$ discharge rate. Arrows indicates the direction of increasing time and plots are made at 20 evenly spaced times between 0 and $0.10 \mathrm{hrs}$. The leading order electrolyte equations are used to evaluate the first order terms in the corrected SP model, see (20). 
(i) a graphite anode $\mathrm{LiC}_{6}$, (ii) an $\mathrm{Li}\left(\mathrm{Ni}_{0.4} \mathrm{Co}_{0.6}\right) \mathrm{O}_{2}$ cathode (nickel-cobalt oxide a variant of NMC) and (iii) an LFP lithium-iron phosphate $\mathrm{LiFePO}_{4}$ cathode. The parameter values for the former two of these are taken from the work of Ecker et. al [14, 15] whilst the latter are taken from Ranom [36]. The open circuit voltages $U_{\text {eq }}\left(c_{s}\right)$ for all three materials are fitted to data from $[14,15,36]$ in figure 3 , while the electrolyte diffusion coefficient $D(c)$ and the electrolyte conductivity $\kappa(c)$, for all three electrodes, is fitted to data in $[14,15]$ in figure 2 (top). Finally the active material diffusion coefficient $D_{s}\left(c_{s}\right)$ for the graphite and NMC particles are fitted to data from $[14,15]$ in figure 2 (bottom); despite the perhaps rather crude fit of $D_{s}\left(c_{s}\right)$ to the GITT data for these two materials the predictions made with these nonlinear diffusion coefficients lead to good fits to the real cell data of [15], as illustrated in figure 8. In a forthcoming work we use more realistic fits to these diffusion coefficients to generate very favourable comparisons between numerical solutions of DFN and real drive cycle data, a much tougher problem than comparing to galvanostatic discharge data. Diffusion in the LFP nanoparticles is modelled by a linear diffusion equation with diffusion coefficient $D_{s}=9 \times 10^{-14} \mathrm{~m}^{2} \mathrm{~s}^{-1}$ as in Table 1 (this is so fast and the particles so small that diffusion is effectively instantaneous). All other parameter values are tabulated in Tables 1 and 2. Figure 5 shows electrolyte variables ( $c$ upper left panel and $\Phi$ upper right panel) plotted as a function of distance $x$ across the half cell in addition to lithium concentration on the particle surfaces $\left.c_{s}\right|_{r=R}$ (lower left panel) and electrode potential $\Phi_{s}$ (lower right panel), all for an $8 \mathrm{C}$ discharge of the $\mathrm{Li}\left(\mathrm{Ni}_{0.4} \mathrm{Co}_{0.6}\right) \mathrm{O}_{2}$ half-cell.

We observe that the basic SP model, reliably reproduces the results of the full DFN model across a range of chemistries provided that the rates are relatively low, i.e., less than $1 \mathrm{C}$ or so. As discharge rates increase beyond around $1 \mathrm{C}$ the accuracy of the SP model deteriorates. However, if the correction terms are accounted for, and the correct SP model is used, the range of accuracy can be expanded significantly. In particular, for graphite, the results of full DFN and the cSP model are almost indistinguishable even up to the relatively aggresive rate of $12 \mathrm{C}$. For NMC and LFP electrodes, excellent agreement between the DFN and cSP model is maintained until around $16 \mathrm{C}$ and $4 \mathrm{C}$ respectively. Beyond these values, even the cSP model begins to be noticeably different from the DFN model, but, still performs far better than the usual SP model approximation. One interesting feature of these comparisons is that the discrepancy between the DFN and cSPM (or cMPM) is greatest, for graphite and LFP, near full discharge. This is because the open circuit voltage curves, for both these materials, is steepest close to full discharge and hence small errors in $c_{s}$ on the electrode particle surfaces can lead to significant errors in the cell voltage.

As we will discuss in more detail in $\S 5$ the reliability of the cSP model is intimately linked to the characteristic size in the change of the overpotential of the electrode material as it is (de)lithiated. In particular it is surprising that good agreement is obtained between the cSP model and the DFN model in the case of the LFP electrode because formally the SP model derivation, as we shall show in $\S 5$, should not apply to a material with such a flat discharge curve. 


\subsection{Comparison between Multiple Particle Model and DFN model}

If instead of an electrode comprised of just one size of electrode particle we consider an electrode composed of two sizes of particle distributed in space so that

$$
R(x)=\left\{\begin{array}{ll}
R^{(a)} & \text { for } \quad 0 \leq x<\alpha \\
R^{(b)} & \text { for } \quad \alpha \leq x<L
\end{array},\right.
$$

we find that the leading order equations (16)-(18) reduce to the following version of the multiparticle (MP) model:

$$
\begin{gathered}
\left.\begin{array}{c}
\frac{\partial c_{s}^{(a)}}{\partial t}=\frac{1}{r^{2}} \frac{\partial}{\partial r}\left(r^{2} D_{s}\left(c_{s}^{(a)}\right) \frac{\partial c_{s}^{(a)}}{\partial r}\right) \quad \text { in } \quad 0 \leq r \leq R^{(a)} \\
c_{s}^{(a)} \text { bounded on } r=0,\left.\quad c_{s}^{(a)}\right|_{r=R^{(a)}}=\mathcal{C}(t)
\end{array}\right\},\left.\quad c_{s}^{(a)}\right|_{t=0}=c_{s, i n i t}, \\
\left.\begin{array}{c}
\frac{\partial c_{s}^{(b)}}{\partial t}=\frac{1}{r^{2}} \frac{\partial}{\partial r}\left(r^{2} D_{s}\left(c_{s}^{(b)}\right) \frac{\partial c_{s}^{(b)}}{\partial r}\right) \quad \text { in } \quad 0 \leq r \leq R^{(b)}, \\
c_{s}^{(b)} \text { bounded on } r=0,\left.\quad c_{s}^{(b)}\right|_{r=R^{(b)}}=\mathcal{C}(t),
\end{array}\right\},\left.\quad c_{s}^{(b)}\right|_{t=0}=c_{s, i n i t}, \\
\left.\int_{0}^{\alpha} b(x)\left(D_{s}\left(c_{s}^{(a)}\right) \frac{\partial c_{s}^{(a)}}{\partial r}\right)\right|_{r=R^{(a)}} d x+\left.\int_{\alpha}^{L} b(x)\left(D_{s}\left(c_{s}^{(b)}\right) \frac{\partial c_{s}^{(b)}}{\partial r}\right)\right|_{r=R^{(b)}} d x=\frac{I(t)}{A F} .
\end{gathered}
$$

Once these have been solved, the functions $j_{n}(x, t)$ and $\mathcal{C}(t)$ can, once again, be used as inputs in the electrolyte model (21)-(25) which we can then solve to obtain the data necessary to compute the voltage according to the cMP model, i.e. via (20). In the multiple particle case the reaction current density, $j_{n}$, has a simple spatial dependence; it is piecewise constant and changes value across $x=\alpha$.

Figures 6 compare the results of this multiple particle model (with 2 particle sizes), both without the correction term (MPmodel) and with the correction term (cMP model), to the DFN model. Here we take $\alpha=L / 2$ and $R^{(a)}=4 R^{(b)}, b^{(a)}=b^{(b)} / 4$ in all three cases while $R^{(b)}$ and $b^{(b)}$ are given by the values of $R_{0}$ and $b_{0}$ in Table 1 for the appropriate chemistries. Figure 7 shows the equivalent electrolyte variables ( $c$ upper left panel and $\Phi$ upper right panel) plotted as a function of distance $x$ across the half cell in addition to lithium concentration on the particle surfaces $\left.c_{s}\right|_{r=R(x)}$ (lower left panel) and electrode potential $\Phi_{s}$ (lower right panel), all for an $8 \mathrm{C}$ discharge of the graded $\mathrm{Li}\left(\mathrm{Ni}_{0.4} \mathrm{Co}_{0.6}\right) \mathrm{O}_{2}$ half-cell.

We note that the inclusion of the first order correction terms into the model (i.e. upgrading to the cMP model) in figure 6 significantly improves the agreement with the full DFN simulation over that with the simple MP model throughout the range of (dis)charge

rates and chemistries we explored. Notably in the case of the LFP half-cell the agreement of cMP model with the DFN simulations is not as good as it was for the electrode with single particle size. However some discrepancy is to be expected between the cMP model and the DFN model for an electrode formed from an active material with such a flat discharge curve. 

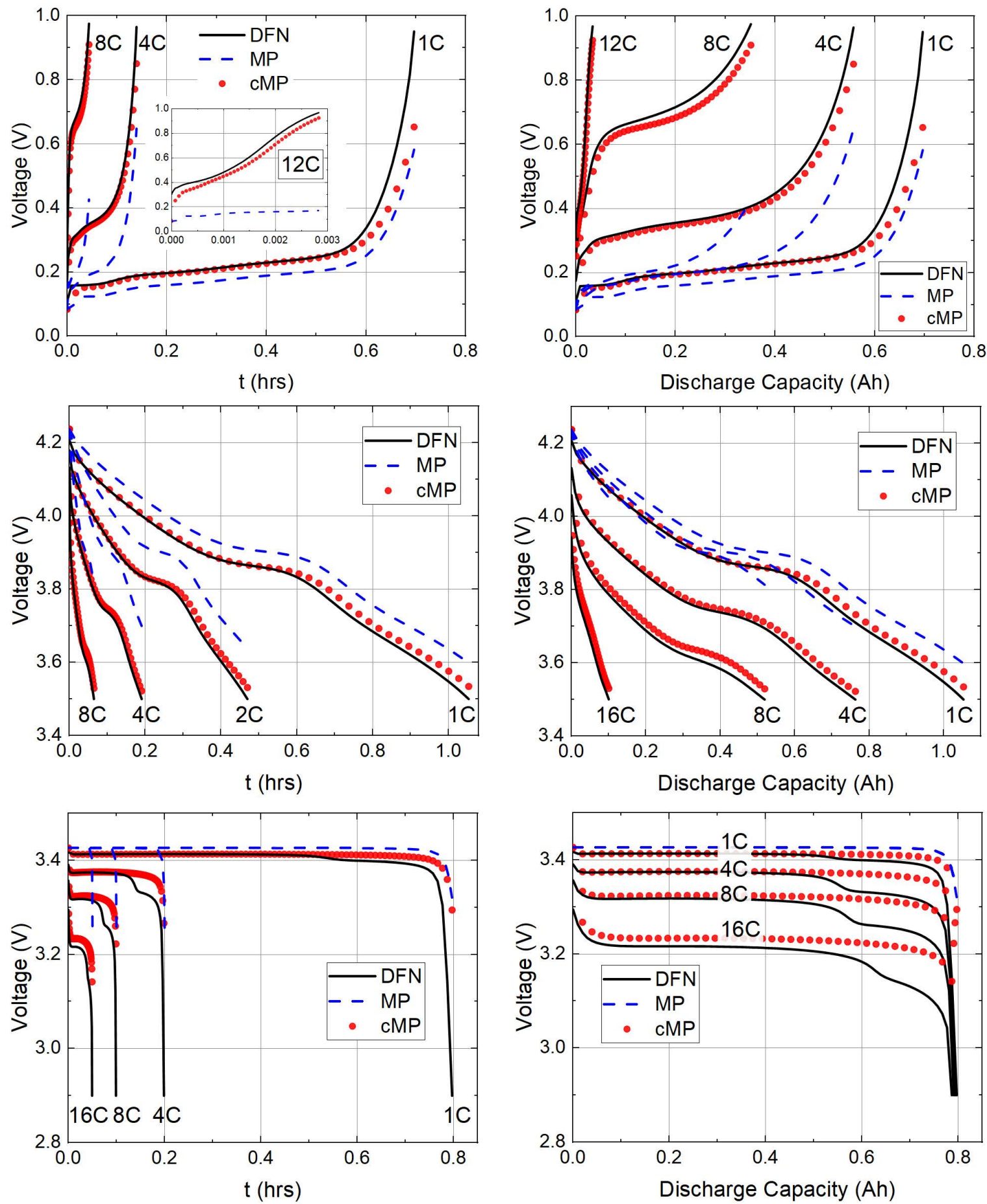

Figure 6: Cell potentials $V$ calculated using the full DFN model, MP model and the cMP model for graphite $\left(\mathrm{LiC}_{6}\right)$ anode (top row), $\mathrm{Li}\left(\mathrm{Ni}_{0.4} \mathrm{Co}_{0.6}\right) \mathrm{O}_{2}$ cathode (middle row) and LFP $(\mathrm{LiFePO})_{4}$ electrode (bottom row) at different discharge rates. Results are shown against both time (left) and discharge capacity (right). 

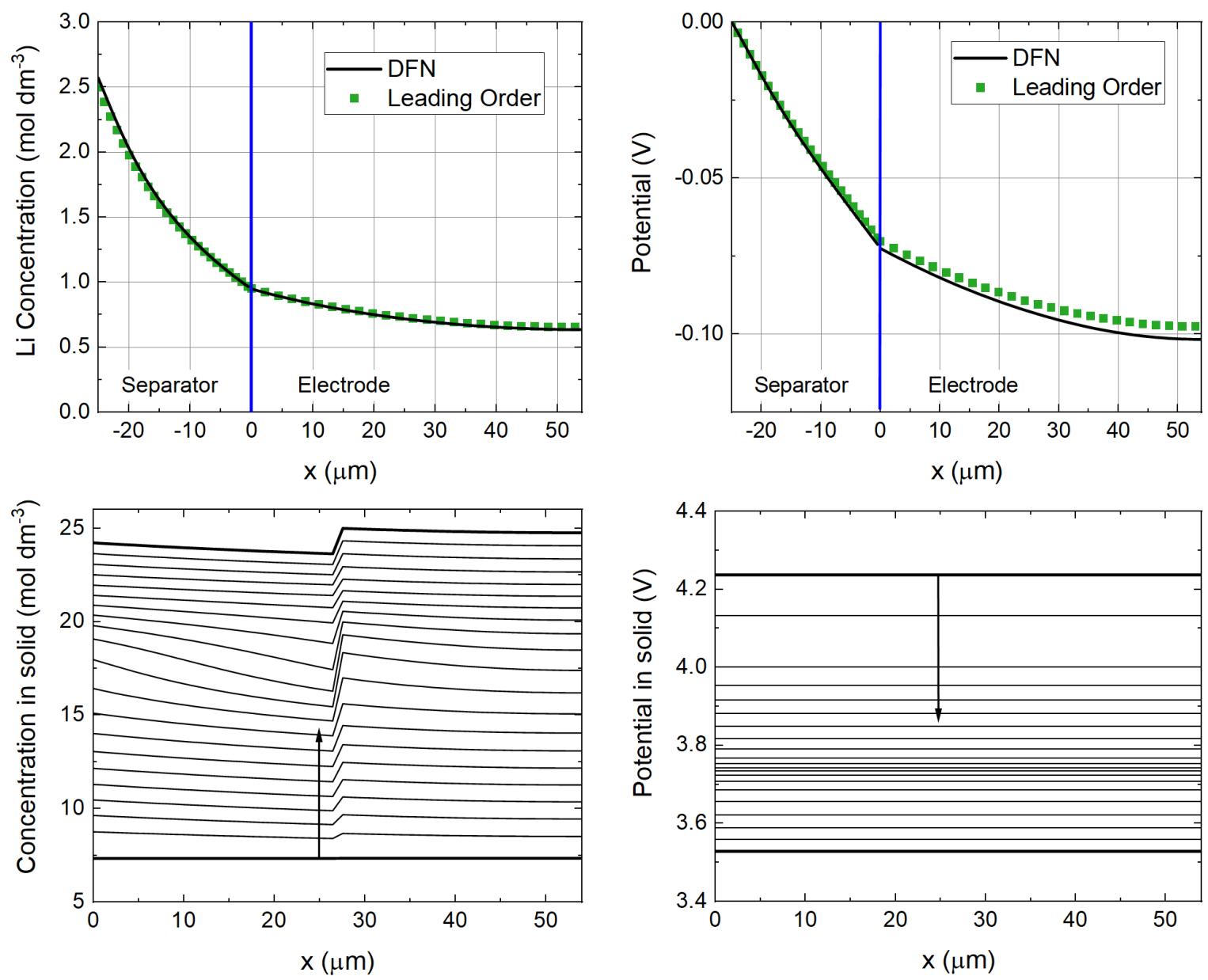

Figure 7: Concentration of $\mathrm{Li}^{+}$ions (top left) and potential (top right) in the electrolyte across a half-cell with an $\mathrm{Li}\left(\mathrm{Ni}_{0.4} \mathrm{Co}_{0.6}\right) \mathrm{O}_{2}$ electrode formed from particles of two different sizes and calculated using the full DFN model and the leading order approximation at $4 \mathrm{C}$ discharge rate. In the upper panels a single snapshot in time (at the end of discharge) is shown because the electrolyte approaches a steady-state rapidly and hence the profiles at earlier times look very similar to those shown. Concentration of $\mathrm{Li}$ on the particle surfaces (bottom left) and potential in solid (bottom right) across an $\mathrm{Li}\left(\mathrm{Ni}_{0.4} \mathrm{Co}_{0.6}\right) \mathrm{O}_{2}$ electrode with two different particle sizes calculated using the full DFN model at $4 \mathrm{C}$ discharge rate. Arrows indicates the direction of increasing time and plots are made at 20 evenly spaced times between 0 and 0.19 hrs. 


\section{Nondimensionalisation of the model}

Before applying asymptotic methods to derive the approximate models described in (16)-(25) from the underlying DFN model (1)-(10) we must non-dimensionalise the governing equations. A key quantity, that will be used later for our temporal scaling, is $\tau$ the characteristic timescale for cell (dis)charge, i.e., the characteristic timescale over which an electrode can sustain a current of size, $\hat{I}$. This is

$$
\tau=\frac{A L F c_{s}^{\max } \hat{b} \hat{R}}{\hat{I}}
$$

where $\hat{I}, \hat{b}$ and $\hat{R}$ are typical sizes of the $I, b$ and $R$ respectively. The timescale $\tau$ can be directly assessed by examining typical C-rates used for battery discharge. Here, we will examine a range of $\mathrm{C}$-rates from the relatively mild $(1 \mathrm{C}$, corresponding to a half cycle time of 1hour) to the relatively aggresive (16C, corresponding to a half cycle time of 3.75 minutes).

The spatial coordinate $x$ is scaled based on the width of the electrode $L$. The quantities $c, c_{s}, R, D, D_{s}, \kappa, I, \mathcal{B}, \sigma$ and $b$ are scaled with their typical values, namely $c_{\text {init }}, c_{s}^{\max }, \hat{R}, \hat{D}$, $\hat{D}_{s}, \hat{\kappa}, \hat{I}, \hat{\mathcal{B}}, \hat{\sigma}$ and $\hat{b}$ respectively. The $\mathrm{BV}$ reaction rate will be scaled based on the average flux required through particle surfaces to sustain a current of size $\hat{I}$. The scaling for the effective ionic flux is based on the size of the diffusive flux carried by a concentration of size $c_{\text {init }}$ over lengths of size $L$ with an effective diffusivity of size $\hat{\mathcal{B}} \hat{D}$.

It remains to specify scalings for the various different potentials. One natural scale is that of the variation of the electric potential within the electrolyte. The conductivities of typical electrolytes are chosen so that only small variations in potential, on the order of the thermal voltage $R_{g} T / F=26 \mathrm{mV}$, are sufficient to carry the requisite current densities suggesting that an appropriate scaling for $\Phi$ is the thermal voltage. A second natural potential scale, which we will henceforth refer to as the characteristic half cell voltage, $\mathcal{U}$, is the size of the difference between the overpotentials of a fully lithiated electrode particle and that of a fully delithiated one. The overpotential of graphite varies between around $0 \mathrm{~V}$ at full lithiation and $1 \mathrm{~V}$ at full delithiation whilst the various metal oxides used in cathodes, e.g. NMC, exhibit variations in their overpotentials of $3 \mathrm{~V}$ at full lithation and $4 \mathrm{~V}$ at full delithitaion. We proceed on the basis that a typical value the characteristic cell potential, $\mathcal{U}$ can be taken to be on the order of $1 \mathrm{~V}$. A notable exception is LFP which exhibits a extremly flat discharge curve for which $\mathcal{U} \leq 26 \mathrm{mV}$. In order that the reaction rates on the particle surfaces are of the requisite size to satitate a current demand of size $\hat{I}$ we should scale both the electrode overpotential, $U_{\text {eq }}$, and the solid electrode potential, $\Phi_{s}$, with the characteristic cell voltage. It is the vast disparity between the thermal voltage and the characteristic cell voltage that gives rise to the large value of the dimensionless parameter $\lambda$ and facilitates the asymptotic analysis that justifies the derivation of the simplified models. In summary, our scalings for 
the problem are

$$
\begin{array}{llll}
t=\tau t^{*} & x=L x^{*} & r=\hat{R} r^{*} & D=\hat{D} D^{*} \\
D_{s}=\hat{D}_{s} D_{s}^{*} & \kappa=\hat{\kappa} \kappa^{*} & c=c_{\text {init }} c^{*} & c_{s}=c_{s}^{\max } c_{s}^{*} \\
V=\mathcal{U} V^{*} & \Phi=\frac{R_{g} T}{F} \Phi^{*} & \Phi_{s}=\mathcal{U} \Phi_{s}^{*} & j=\frac{\hat{I}}{A} j^{*} \\
j_{s}=\frac{\hat{I}}{A} j_{s}^{*} & I=\hat{I} I^{*} & j_{n}=\frac{\hat{I}}{A L \hat{b}} j_{n}^{*} & \eta=\frac{R_{g} T}{F} \eta^{*} \\
U_{\text {eq }}=\mathcal{U} U_{\text {eq }}^{*} & N_{-}=\frac{\hat{\mathcal{B}} \hat{D} c_{\text {init }}}{L} N_{-}^{*} & \mathcal{B}=\hat{\mathcal{B} \mathcal{B}^{*}} & b=\hat{b} b^{*} \\
I=\hat{I} I^{*} & R=\hat{R} R^{*} & \sigma=\hat{\sigma} \sigma^{*} . &
\end{array}
$$

The non-dimensionalisation gives rise to the following dimensionless quantities that characterise the system:

$$
\begin{array}{llll}
\mathcal{N}=\frac{L^{2}}{\tau \hat{\mathcal{B}} \hat{D}}, & \Gamma=\frac{\hat{I} L}{A c_{\text {init }} \hat{D} \hat{\mathcal{B}} F}, & \lambda=\frac{\mathcal{U} F}{R_{g} T}, & \mathcal{Q}=\frac{\hat{R}^{2}}{\tau \hat{D}_{s}}, \\
\Upsilon=\frac{k c_{s, \text { init }}{ }^{1 / 2} c_{s}^{\max } A L F \hat{b}}{\hat{I}}, & \Theta=\frac{\hat{\sigma} R_{g} T A}{L \hat{I} F}, & \mathcal{P}=\frac{\hat{\mathcal{B}} \hat{\kappa} R_{g} T A}{L \hat{I} F}, & \mathcal{L}_{s}=\frac{L_{s}}{L} \\
\bar{c}_{\mathrm{s}, \text { init }}=\frac{c_{s, \text { init }}}{c_{s}^{\text {max }}} . & &
\end{array}
$$

Of those parameters whose meaning is not self-evident from their definition; $\mathcal{N}$ is the timescale for diffusion in the electrolyte over the timescale for cell discharge, $\Gamma$ is the drift flux over the diffusive flux, $\mathcal{Q}$ is the timescale for diffusive transport inside the electrode particles over the timescale for cell discharge, $\Upsilon$ is the total amount of lithium intercalated into the active material per second over the current, $\Theta$ is the electronic conductivity of the solid scaffold over the characteristic conductivity of the electrode material, $\mathcal{P}$ is the ionic conductivity over the characteristic conductivity of the electrode material.

\subsection{The Half-Cell Dimensionless Model}

On dropping the stars from the dimensionless variables the dimensionless problem reads as follows. In the region $-\mathcal{L}_{s}<x<1$ the (dimensionless) electrolyte equations are

$$
\begin{aligned}
& \epsilon_{l}(x) \mathcal{N} \frac{\partial c}{\partial t}+\frac{\partial N_{-}}{\partial x}=0, \quad N_{-}=-\mathcal{B}(x) D(c) \frac{\partial c}{\partial x}-\Gamma\left(1-t^{+}\right) j, \\
& \frac{\partial j}{\partial x}=\left\{\begin{array}{llc}
0 & \text { for } & -\mathcal{L}_{s}<x<0 \\
b(x) j_{n}(x, t) & \text { for } & 0<x<1
\end{array},\right. \\
& j=-\mathcal{P} \kappa(c) \mathcal{B}(x)\left(\frac{\partial \Phi}{\partial x}-2 \frac{1-t^{+}}{c} \frac{\partial c}{\partial x}\right),
\end{aligned}
$$

and satisfy the boundary conditions

$$
\left.j\right|_{x=-\mathcal{L}_{s}}=I(t),\left.\quad N_{-}\right|_{x=-\mathcal{L}_{s}}=0,\left.\quad \Phi\right|_{x=-\mathcal{L}_{s}}=0,\left.\quad N_{-}\right|_{x=1}=0 .
$$


Table 1: Parameter values for different chemistries based on a $1 \mathrm{C}$ discharge rate.

\begin{tabular}{|c|c|c|c|c|}
\hline Parameter & Units & $\begin{array}{c}\text { Graphite }\left(\mathrm{LiC}_{6}\right) \\
{[15,14]}\end{array}$ & $\begin{array}{c}\mathrm{Li}\left(\mathrm{Ni}_{0.4} \mathrm{Co}_{0.6}\right) \mathrm{O}_{2} \\
{[15,14]}\end{array}$ & $\begin{array}{c}\mathrm{LFP}\left(\mathrm{LiFePO}_{4}\right) \\
{[36,48]}\end{array}$ \\
\hline Electrode thickness, $L$ & $\mu \mathrm{m}$ & 74 & 54 & 62 \\
\hline Electrode particle radius, $R_{0}$ & $\mu \mathrm{m}$ & 13.7 & 6.5 & 0.05 \\
\hline Electrode cross-section area, $A$ & $\mathrm{~m}^{2}$ & $8.585 \times 10^{-3}$ & $8.585 \times 10^{-3}$ & $10^{-4}$ \\
\hline Vol. fraction electrolyte, $\epsilon_{l}$ & - & 0.329 & 0.296 & 0.4764 \\
\hline $\begin{array}{c}\text { Brunauer-Emmett-Teller } \\
\text { surface area, } b_{0}=3\left(1-\epsilon_{l}\right) / R_{0}\end{array}$ & $m^{-1}$ & $1.469 \times 10^{5}$ & $3.249 \times 10^{5}$ & $3.142 \times 10^{7}$ \\
\hline Conductivity in solid, $\sigma_{0}$ & $\mathrm{~S} \mathrm{~m}^{-1}$ & 14.0 & 68.1 & 0.5 \\
\hline $\begin{array}{l}\text { Permeability factor of } \\
\text { electrolyte, } \mathcal{B}_{0}\end{array}$ & - & 0.162 & 0.153 & 0.329 \\
\hline Reaction rate constant, $k$ & $\mathrm{~m}^{2.5} \mathrm{~s}^{-1} \mathrm{~mol}^{-0.5}$ & $2.333 \times 10^{-10}$ & $5.904 \times 10^{-11}$ & $3 \times 10^{-12}$ \\
\hline $\begin{array}{l}\text { Maximum concentration of } \\
\mathrm{Li} \text { ions in solid, } c_{s}^{\max }\end{array}$ & $\mathrm{mol} \mathrm{m}^{-3}$ & 17715.6 & 28176.4 & 18805 \\
\hline Transference number, $t^{+}$ & - & 0.26 & 0.26 & 0.3 \\
\hline $1 \mathrm{C}$ current draw, $I_{0}$ & A & -0.15625 & 0.15625 & 0.0015 \\
\hline Contact resistance, $R_{c}$ & $\Omega$ & 0 & 0 & 0 \\
\hline Absolute temperature, $T$ & $K$ & 298.15 & 298.15 & 298.15 \\
\hline $\begin{array}{l}\text { Typical concentration of } \mathrm{Li} \\
\text { in liquid, } c_{\text {init }}\end{array}$ & $\mathrm{mol} \mathrm{m}^{-3}$ & 1000 & 1000 & 1000 \\
\hline Typical diffusivity liquid, $D_{0}$ & $\mathrm{~m}^{2} \mathrm{~s}^{-1}$ & $2.594 \times 10^{-10}$ & $2.594 \times 10^{-10}$ & $2.594 \times 10^{-10}$ \\
\hline Typical conductivity liquid, $\kappa_{0}$ & $\mathrm{~S} \mathrm{~m}^{-1}$ & 1.0 & 1.0 & 1.0 \\
\hline Diffusivity in solid, $D_{s}$ & $\mathrm{~m}^{2} \mathrm{~s}^{-1}$ & Fig.2 & Fig. 2 & $9 \times 10^{-14}$ \\
\hline Typical diffusivity in solid, $\mathcal{D}_{0}$ & $\mathrm{~m}^{2} \mathrm{~s}^{-1}$ & $3 \times 10^{-14}$ & $10^{-13}$ & $9 \times 10^{-14}$ \\
\hline Equilibrium potential, $U_{\text {eq }}$ & $\mathrm{V}$ & Fig.3 & Fig. 3 & Fig.3 \\
\hline Characteristic cell voltage, $\mathcal{U}$ & V & 0.2 & 1.0 & 0.0257 \\
\hline Discharge time scale $(32), \tau$ & $\mathrm{s}$ & $1.399 \times 10^{4}$ & $1.703 \times 10^{4}$ & $1.178 \times 10^{4}$ \\
\hline \multicolumn{5}{|c|}{ Derived dimensionless quantities (39)-(41) } \\
\hline $\mathcal{N}=L^{2} /(\tau \hat{\mathcal{B}} \hat{D})$ & - & 0.0093 & 0.0043 & 0.0038 \\
\hline$\Gamma=I_{0} L /\left(A c_{\text {init }} \hat{D} \hat{\mathcal{B}} F\right)$ & - & 0.332 & 0.257 & 0.113 \\
\hline$\Upsilon=k c_{\text {init }}^{1 / 2} c_{s}^{\max } A L F \hat{b} / \hat{I}$ & - & 7.53 & 4.89 & 22.4 \\
\hline$\Theta=\hat{\sigma} R_{g} T A /(L \hat{I} F)$ & - & 267 & 1780 & 13.8 \\
\hline $\mathcal{P}=\hat{\mathcal{B}} \hat{\kappa} R T A /(L \hat{I} F)$ & - & 3.1 & 4.0 & 9.1 \\
\hline $\mathcal{Q}=\hat{R}^{2} / \tau \hat{D}_{s}$ & - & 0.447 & 0.0248 & $2.36 \times 10^{-6}$ \\
\hline$\lambda=\mathcal{U} F / R_{g} T$ & - & 7.78 & 38.92 & 1.00 \\
\hline
\end{tabular}


Table 2: Parameters of the separator used in the model

\begin{tabular}{|c|c|c|}
\hline Parameter & Units & Separator [42] \\
\hline Thickness, $L^{\text {sep }}$ & $\mu \mathrm{m}$ & 25 \\
Volume fraction of electrolyte, $\epsilon_{l}^{\text {sep }}$ & - & 0.55 \\
Permeability factor of electrolyte, $\mathcal{B}_{0}^{\text {sep }}$ & - & 0.408 \\
\hline
\end{tabular}

These couple to the (dimensionless) electrode equations in the region $0<x<1$

$$
\begin{array}{r}
j_{s}=-\lambda \Theta \sigma \frac{\partial \Phi_{s}}{\partial x}, \quad \frac{\partial j_{s}}{\partial x}=-b(x) j_{n}(x, t), \\
j_{n}(x, t)=2 \Upsilon c^{1 / 2}\left(\left.c_{s}\right|_{r=R(x)}\right)^{1 / 2}\left(1-\left.c_{s}\right|_{r=R(x)}\right)^{1 / 2} \sinh \left(\frac{\eta}{2}\right), \\
\eta=\lambda\left(\Phi_{s}-U_{\text {eq }}\left(\left.c_{s}\right|_{r=R(x)}\right)\right)-\Phi, \\
\mathcal{Q} \frac{\partial c_{s}}{\partial t}=\frac{1}{r^{2}} \frac{\partial}{\partial r}\left(r^{2} D_{s}\left(c_{s}\right) \frac{\partial c_{s}}{\partial r}\right), \quad \text { for } \quad 0 \leq r \leq R(x), \\
c_{s} \text { bounded on } r=0, \quad-\left.D_{s} \frac{\partial c_{s}}{\partial r}\right|_{r=R(x)}=\mathcal{Q} j_{n} .
\end{array}
$$

which in turn satisfy two boundary conditions in $x$, namely

$$
\left.j_{s}\right|_{x=0}=0,\left.\quad j_{s}\right|_{x=1}=I(t), \quad V(t)=\left.\Phi_{s}\right|_{x=1} .
$$

The final condition in (51) serves to determine the half-cell voltage $V(t)$ from the solution to the problem. Initial conditions corresponding to a half-cell which is initially at equilibrium are

$$
\left.c\right|_{t=0}=1,\left.\quad c_{s}\right|_{t=0}=\bar{c}_{\mathrm{s}, \text { init }} .
$$

where $\bar{c}_{\mathrm{s}, \text { init }}$ is uniform throughout the cell (i.e. is independent of both $x$ and $r$ ).

Features of the model Some helpful features of the model can be derived by taking the sum of equations (43) and (46b) in the region $0<x<1$, integrating the results and applying the boundary conditions $(45 \mathrm{~d})$ and $(51 \mathrm{~b})$. We arrive at

$$
j+j_{s}=I(t)
$$

Thus, the total (both ionic and electronic) current density is uniform throughout the electrode. Furthermore, on integrating (43) through the thickness of the electrode $\left(-\mathcal{L}_{s}<x<1\right)$ and applying the boundary conditions (45a) and (51b) we obtain the integral condition

$$
\int_{0}^{1} b(x) j_{n}(x) d x=-I(t) .
$$

This amounts to the observation that the total intercalating current flowing into the electrode is equal to the current flowing from the electrode into the current collector and around the circuit. 


\section{Asymptotic reduction: derivation of single and mul- tiple particle models}

In this section we systematically derive both the basic SP model and the cSP model for the half cell from the dimensionless DFN model (42)-(51) using asymptotic methods in the large $\lambda$ limit. In addition to deriving these SP models we also derive natural extensions that describe electrodes with more than one particle size and/or chemistry. This analysis can easily be extended to a full-cell, with two porous electrodes, but we do not do this here, instead relegating this result to Appendix B. Formally we investigate the distinguished limit in which $\lambda \rightarrow \infty$ and all other dimensionless parameters (i.e. $\mathcal{N}, \Gamma, \mathcal{L}_{s}, \mathcal{P}, \Theta, \Upsilon, \bar{c}_{\mathrm{s} \text {,init }}$ and $\mathcal{Q}$ ) are of order 1 . Note that this means that the results we derive on this basis have a wider validity than the formal assumption that all parameters, with the exception of $\lambda$, are $O(1)$. In particular we will subsequently consider the small $\mathcal{Q}$ limit, after first having taken the large $\lambda$ limit, in order to derive an approximate model for a graded electrode with a spatial distribution of particle sizes. We note that while the large $\lambda$ limit would seem to provide a good description of electrodes fabricated from the active materials graphite (for which $\lambda \approx 7.8$ ) and $\mathrm{Li}\left(\mathrm{Ni}_{0.4} \mathrm{Co}_{0.6}\right) \mathrm{O}_{2}$ (for which $\lambda \approx 38.9$ ) it would not be expected to work particularly well for LFP (for which $\lambda \approx 1$ ).

The key observation that leads to the SP model is that in order that the dimensionless current $I(t)$ is of size $O(1)$ the total amount of intercalation throughout the electrode must also be size $O(1)$ (see (54)). In turn this requires that, the dimensionless overpotential, $\eta$ is $O(1)$ throughout the electrode which leads to the condition that

$$
\Phi-\lambda\left(\Phi_{s}-U_{\text {eq }}\left(\left.c_{s}\right|_{r=R(x)}\right)\right)=O(1) .
$$

Since $I(t)$ is $O(1)$ it follows that both the dimensionless current densities $j$ and $j_{s}$, within the electrolyte and electrode respectively, are $O(1)$. The condition that $j=O(1)$ and equation (45) imply that $\Phi=O(1)$ while the condition that $j_{s}=O(1)$ means that gradients in $\Phi_{s}$ are $O(1 / \lambda)$. This leads us to the following asymptotic expansion:

$$
\begin{array}{ccc}
\Phi_{s}=\Phi_{s, 0}+\frac{1}{\lambda} \Phi_{s, 1}+\cdots, & V=V_{0}(t)+\frac{1}{\lambda} V_{1}(t)+\cdots, & c_{s}=c_{s, 0}+\frac{1}{\lambda} c_{s, 1} \cdots, \\
j_{s}=j_{s, 0}+\frac{1}{\lambda} j_{s, 1} \cdots, & \eta=\eta_{0}+\frac{1}{\lambda} \eta_{1} \cdots, & j_{n}=j_{n, 0}+\frac{1}{\lambda} j_{n, 1} \cdots, \\
\Phi=\Phi_{0}+\cdots, & c=c_{0}+\cdots & j=j_{0}+\cdots,
\end{array}
$$

\subsection{The leading order problem}

The derivation of the leading order term presented here broadly follows that in the thesis of Ranom [36]. On inserting the expansions (56) into the equations (46)-(51) and taking the 
leading order terms we obtain the following problem in $0 \leq x \leq 1$ :

$$
\begin{aligned}
\frac{\partial \Phi_{s, 0}}{\partial x} & =0, \\
\frac{\partial j_{s, 0}}{\partial x} & =-b(x) j_{n, 0}(x, t),\left.\quad j_{s, 0}\right|_{x=0}=0,\left.\quad j_{s, 0}\right|_{x=1}=I(t), \\
\Phi_{s, 0}= & U_{\text {eq }}\left(\left.c_{s, 0}\right|_{r=R(x)}\right), \quad V_{0}(t)=\left.\Phi_{s, 0}\right|_{x=1}, \\
\mathcal{Q} \frac{\partial c_{s, 0}}{\partial t}= & \frac{1}{r^{2}} \frac{\partial}{\partial r}\left(r^{2} D_{s}\left(c_{s, 0}\right) \frac{\partial c_{s, 0}}{\partial r}\right) \quad \text { in } \quad 0 \leq r \leq R(x), \\
c_{s, 0} & \text { bounded on } r=0,\left.\quad D_{s}\left(c_{s, 0}\right) \frac{\partial c_{s, 0}}{\partial r}\right|_{r=R(x)}=-\mathcal{Q} j_{n, 0}(x, t), \\
\left.c_{s, 0}\right|_{t=0}= & \bar{c}_{\mathrm{s}, \text { init }} .
\end{aligned}
$$

This system is solved by noting that the integral of (57) implies that $\Phi_{s, 0}=\Phi_{s, 0}(t)$ and in turn that (59a) implies that

$$
\left.c_{s, 0}\right|_{r=R(x)}=U_{\mathrm{eq}}^{-1}\left(\Phi_{s, 0}(t)\right) .
$$

Integrating (58a) between $x=0$ and $x=1$ and applying the boundary conditions (58b)-(58c) leads to the condition

$$
\int_{0}^{1} b(x) j_{n, 0}(x, t) d x=-I(t)
$$

which is equivalent to the leading order term of the condition (54). The leading order problem thus comprises the sequence of electrode particle problems (60)-(62) coupled to the conditions (63)-(64) with the leading order half-cell potential $V_{0}(t)$ being found from $(59 \mathrm{~b})$. In summary the leading order problem can be written in the form

$$
\begin{array}{r}
\mathcal{Q} \frac{\partial c_{s, 0}}{\partial t}=\frac{1}{r^{2}} \frac{\partial}{\partial r}\left(r^{2} D_{s}\left(c_{s, 0}\right) \frac{\partial c_{s, 0}}{\partial r}\right) \quad \text { in } \quad 0 \leq r \leq R(x), \\
c_{s, 0} \text { bounded on } r=0,\left.\quad c_{s, 0}\right|_{r=R(x)}=\mathcal{C}_{0}(t), \\
\left.\int_{0}^{1} b(x)\left(D_{s}\left(c_{s, 0}\right) \frac{\partial c_{s, 0}}{\partial r}\right)\right|_{r=R(x)} d x=\mathcal{Q} I(t), \\
V_{0}(t)=\Phi_{s, 0}(t)=U_{\mathrm{eq}}\left(\mathcal{C}_{0}(t)\right) .
\end{array}
$$

Here $\mathcal{C}_{0}(t)$ must be chosen so that the integral condition (67) is satisfied. The leading order reaction rate $j_{n, 0}(x, t)$ is determined from the solution to this problem via the condition

$$
j_{n, 0}(x, t)=-\left.\frac{1}{\mathcal{Q}} D_{s}\left(c_{s, 0}\right) \frac{\partial c_{s, 0}}{\partial r}\right|_{r=R(x)} .
$$

Remark. The solution of the sequence of diffusion problems (65)-(68) does not represent a significant computational saving if the electrode particle radii $R(x)$ vary continuously in $x$. However where $R(x)$ is piecewise constant a major saving in computational time, over the DFN model, is achieved because, rather than solving a continuum of diffusion problems (65)-(66) in $x$ we need only solve a finite number of such problems. 


\subsubsection{The single particle (SP) model}

In the case of a uniform particle size throughout the half cell the leading order equations simplify significantly because all the particles are identical and so, on writing $R=1$ (recalling that we have nondimensionalised $r$ with typical particle radius $\hat{R}$ ), equations (65)-(68) simplify to

$$
\begin{aligned}
\mathcal{Q} \frac{\partial c_{s, 0}}{\partial t}=\frac{1}{r^{2}} \frac{\partial}{\partial r}\left(r^{2} D_{s}\left(c_{s, 0}\right) \frac{\partial c_{s, 0}}{\partial r}\right) \quad \text { in } \quad 0 \leq r \leq 1, \\
c_{s, 0} \text { bounded on } r=0,\left.\quad\left(D_{s}\left(c_{s, 0}\right) \frac{\partial c_{s, 0}}{\partial r}\right)\right|_{r=1}=\frac{\mathcal{Q} I(t)}{\int_{0}^{1} b(x) d x}, \\
\left.c_{s, 0}\right|_{t=0}=\bar{c}_{\mathrm{s}, \text { nit }}, \quad \text { and } \quad V_{0}(t)=U_{\mathrm{eq}}\left(\left.c_{s, 0}\right|_{r=1}\right) .
\end{aligned}
$$

\subsection{The first order correction term}

Here we seek to calculate the first order correction $V_{1}(t)$ to the voltage across the half-cell. We start by deriving an expression for the first order electrode potential $\Phi_{s, 1}$. Substituting expansion (56) into (46) yields the problem

$$
\frac{\partial \Phi_{s, 1}}{\partial x}=-\frac{j_{s, 0}(x, t)}{\Theta \sigma(x)},\left.\quad \Phi_{s, 1}\right|_{x=1}=V_{1}(t) .
$$

and on solving for $j_{s, 0}$ from (58) and for $\Phi_{s, 1}$ from (73) we obtain the required expression

$$
j_{s, 0}(x, t)=-\int_{0}^{x} b\left(x^{\prime}\right) j_{n, 0}\left(x^{\prime}, t\right) d x^{\prime}, \quad \Phi_{s, 1}(x, t)=V_{1}(t)+\int_{x}^{1} \frac{j_{s, 0}\left(x^{\prime}, t\right)}{\Theta \sigma\left(x^{\prime}\right)} d x^{\prime} .
$$

Notably this formula for $\Phi_{s, 1}$ depends upon $V_{1}(t)$, the quantity that we seek.

Solvability condition. We obtain a solvability condition, that may be used to determine $V_{1}(t)$, by writing down the first order expansion of the electrode current conservation equation (46b) and its boundary conditions (51a-b):

$$
\frac{\partial j_{s, 1}}{\partial x}=-b(x) j_{n, 1}(x, t),\left.\quad j_{s, 1}\right|_{x=0}=0,\left.\quad j_{s, 1}\right|_{x=1}=0 .
$$

As has also been observed in [21, 26], integration of this equation, between $x=0$ and $x=1$, and application of the boundary conditions leads to the solvability condition

$$
\int_{0}^{1} b(x) j_{n, 1}(x, t) d x=0
$$

which is equivalent to the first order terms in (54). It remains to determine an appropriate expression for $j_{n, 1}$ that can be substituted into the solvability condition (76). This is accomplished by substituting the expansion (56) into the boundary condition (50b) and proceeding 
to first order; a procedure that yields the result

$$
j_{n, 1}(x, t)=-\left.\frac{1}{\mathcal{Q}} \frac{\partial}{\partial r}\left(D_{s}\left(c_{s, 0}\right) c_{s, 1}\right)\right|_{r=R(x)}
$$

In order to determine the right-hand side of this expression we need to solve the first order microscopic transport equations inside the electrode particles with an appropriate Dirichlet boundary condition.

A Dirichlet boundary condition for $c_{s, 1}$ on $r=R(x)$. The Dirichlet condition on $c_{s, 1}$ on the electrode particle surfaces, $r=R(x)$, is found by expanding (48) to first order and rearranging the resulting expression to obtain

$$
\left.c_{s, 1}\right|_{r=R(x)}=\mathcal{C}_{1}(x, t) \quad \text { where } \quad \mathcal{C}_{1}(x, t)=\frac{\Phi_{s, 1}(x, t)-\eta_{0}(x, t)-\Phi_{0}(x, t)}{U_{e q}^{\prime}\left(\left.c_{s, 0}\right|_{r=R(x)}\right)} .
$$

In turn, an expression for $\eta_{0}$ may be found from the leading order expansion of (47)

$$
\eta_{0}(x, t)=2 \operatorname{arcsinh}\left(\frac{j_{n, 0}(x, t)}{2 \Upsilon\left[\left.c_{s, 0}\right|_{r=R(x)}\left(1-\left.c_{s, 0}\right|_{r=R(x)}\right) c_{0}(x, t)\right]^{1 / 2}}\right) .
$$

The leading order electrolyte problem for $c_{0}$ and $\Phi_{0}$. In the expression (78) both $\mathcal{C}_{1}(x, t)$ and $\eta_{0}$ depend on the leading order solution in the electrolyte. On substituting the expansion (56) into (42)-(45) we see that this satisfies the following problem:

$$
\begin{aligned}
& \epsilon_{l}(x) \mathcal{N} \frac{\partial c_{0}}{\partial t}+\frac{\partial N_{-, 0}}{\partial x}=0, \quad N_{-, 0}=-\mathcal{B}(x) D\left(c_{0}\right) \frac{\partial c_{0}}{\partial x}-\Gamma\left(1-t^{+}\right) j_{0}, \\
& \frac{\partial j_{0}}{\partial x}=\left\{\begin{array}{llc}
0 & \text { for } & -\mathcal{L}_{s}<x<0 \\
b(x) j_{n, 0}(x, t) & \text { for } & 0<x<1
\end{array},\right. \\
& j_{0}=-\mathcal{P} \kappa\left(c_{0}\right) \mathcal{B}(x)\left(\frac{\partial \Phi_{0}}{\partial x}-2 \frac{1-t^{+}}{c_{0}} \frac{\partial c_{0}}{\partial x}\right), \\
& \left.j_{0}\right|_{x=-\mathcal{L}_{s}}=I(t),\left.\quad N_{-, 0}\right|_{x=-\mathcal{L}_{s}}=0,\left.\quad \Phi_{0}\right|_{x=-\mathcal{L}_{s}}=0,\left.\quad N_{-, 0}\right|_{x=1}=0,\left.\quad c_{0}\right|_{t=0}=1 .
\end{aligned}
$$

The first order problem for lithium transport in the electrode. Substituting the expansion (56) into the microscopic transport equations (49)-(50a) and appending the Dirichilet boundary condition (78) leads to the following problems for $c_{s, 1}(r, x, t)$

$$
\begin{aligned}
\mathcal{Q} \frac{\partial c_{s, 1}}{\partial t}= & \frac{1}{r^{2}} \frac{\partial}{\partial r}\left(r^{2} \frac{\partial}{\partial r}\left(D_{s}\left(c_{s, 0}\right) c_{s, 1}\right)\right) \quad \text { in } \quad 0 \leq r \leq R(x), \\
c_{s, 1} \quad & \text { bounded on } r=0,\left.\quad c_{s, 1}\right|_{r=R(x)}=\mathcal{C}_{1}(x, t),\left.\quad c_{s, 1}\right|_{t=0}=0,
\end{aligned}
$$

where $\mathcal{C}_{1}(x, t)$ is defined in (78) and the initial condition comes from expanding the initial conditions (52). By solving this problem we can find an expression for $\left.\frac{\partial}{\partial r}\left(D_{s}\left(c_{s, 0}\right) c_{s, 1}\right)\right|_{r=R(x)}$, as a function of $\mathcal{C}_{1}(x, t)$, that we can use to determine $j_{n, 1}(x, t)$ using $(77)$. 
Solution of the first order lithium transport problem in the electrode and determing $V_{1}$. The problem (84)-(85) for $c_{s, 1}$ is linear. It can be shown using a Green's function approach that the quantity we seek satisfies an integral equation of the form

$$
\left.\frac{\partial}{\partial r}\left(D_{s}\left(c_{s, 0}\right) c_{s, 1}\right)\right|_{r=R(x)}=\int_{0}^{t} \mathcal{C}_{1}(x, \tau) \mathcal{G}(x, t ; \tau) d \tau,
$$

where the problem for the 'Green's function' $\mathcal{G}(x, t ; \tau)$ is derived in Appendix A. In practice deriving this Green's function for a uniformly varying $R(x)$ is extremely costly and arguably more effort that solving the original DFN model. However in the notable case of a uniform particle size throughout the half-cell, i.e. $R(x) \equiv 1$ the 'Green's function' is independent of $x$ so that in this case

$$
\left.\frac{\partial}{\partial r}\left(D_{s}\left(c_{s, 0}\right) c_{s, 1}\right)\right|_{r=1}=\int_{0}^{t} \mathcal{C}_{1}(x, \tau) \mathcal{G}(t ; \tau) d \tau \quad \text { for a uniform electrode }
$$

Substituting (86) into (77) and the resulting expression back into the solvability condition (76) leads to the result

$$
\int_{0}^{1} b(x) \int_{0}^{t} \mathcal{C}_{1}(x, \tau) \mathcal{G}(x, t ; \tau) d \tau d x=0 .
$$

which provides an avenue to calculate $V(t)$ if we could solve for the Green's function $\mathcal{G}(x, t ; \tau)$.

\subsubsection{First order correction to the Single Particle Model}

One special case where this result is useful is that of uniform particle size throughout the half cell, i.e. $R(x) \equiv 1$ where $\mathcal{G}(x, t ; \tau)=\mathcal{G}(t ; \tau)$. In this instance the spatial integral can be separated from the temporal integral in (88) leading to the simplified condition

$$
\int_{0}^{1} b(x) \mathcal{C}_{1}(x, \tau) d x=0 .
$$

On recalling that $U_{e q}\left(\left.c_{s, 0}\right|_{r=R(x)}\right)$ is a function of $t$ only and substituting for $\mathcal{C}_{1}(x, t)$ (from (78)) and for $\Phi_{s, 1}$ (from (74)) in the above integral we find, after some rearrangement, the following formula for the first order correction to the cell voltage:

$$
V_{1}(t)=\frac{\int_{0}^{1} b(x)\left[\eta_{0}(x, t)+\Phi_{0}(x, t)-\int_{x}^{1} \frac{j_{s, 0}\left(x^{\prime}, t\right)}{\Theta \sigma\left(x^{\prime}\right)} d x^{\prime}\right] d x}{\left(\int_{0}^{1} b(x) d x\right)} .
$$

Here $\eta_{0}(x, t)$ is given by $(79)$ and $j_{s, 0}(x, t)$ by $(74)$. This completes the derivation of the dimensionless cSP model, the dimensional version of which is given in (16)-(18) and (20)$(25)$. 


\subsubsection{First order correction for graded electrodes in small current limit $\mathcal{Q} \ll 1$}

In the case where particle size is non-uniform (i.e. $R(x)$ is non constant) we can still make progress in determining the voltage correction if the current is relatively small, corresponding to a small value of $\mathcal{Q}$. Here we seek series solutions for $c_{s, 0}(r, x, t)$ and $c_{s, 1}(r, x, t)$ in powers of $Q$ to (60)-(62) and (84)-(85), respectively. We find that $c_{s, 0}(r, x, t)$ has an expansion in $Q$ of the form

$$
\begin{aligned}
c_{s, 0}(r, x, t) & =\psi(x, t)+\frac{\mathcal{Q} j_{n, 0}(x, t)}{2 D_{s}(\psi) R(x)}\left(R^{2}(x)-r^{2}\right)+\cdots, \\
\text { where } \quad \frac{\partial \psi}{\partial t} & =-\frac{3 j_{n, 0}(x, t)}{R(x)} .
\end{aligned}
$$

It follows from (84)-(85) that

$$
c_{s, 1}(r, x, t)=\mathcal{C}_{1}(x, t)-\mathcal{Q}\left(\frac{R^{2}(x)-r^{2}}{6 D_{s}(\psi)}\left(\frac{\partial \mathcal{C}_{1}}{\partial t}-\mathcal{C}_{1} \frac{D_{s}^{\prime}(\psi)}{D_{s}(\psi)} \frac{\partial \psi}{\partial t}\right)\right)+\cdots,
$$

and hence the term $D_{s}\left(c_{s, 0}\right) c_{s, 1}$ that appears in the right-hand side of (84) has the expansion

$$
D_{s}\left(c_{s, 0}\right) c_{s, 1}=D_{s}(\psi(x, t)) \mathcal{C}_{1}(x, t)+Q \frac{\partial \mathcal{C}_{1}}{\partial t}\left(\frac{r^{2}-R^{2}(x)}{6}\right)+O\left(\mathcal{Q}^{2}\right) .
$$

Substituting the above into (77) leads to the follows expression for $j_{n, 1}(x, t)$ :

$$
j_{n, 1}(x, t)=-\frac{R(x)}{3} \frac{\partial \mathcal{C}_{1}}{\partial t}+O(\mathcal{Q}) .
$$

Substitution of this expansion into the solvability condition (76) and integrating the result with respect to time yields the integral conditon

$$
\int_{0}^{1} b(x) R(x)\left(\Phi_{s, 1}(x, t)-\Phi_{0}(x, t)-\eta_{0}(x, t)\right) d x=O(\mathcal{Q}) .
$$

On substituting the expression that we have obtained for $\Phi_{s, 1}(x, t)$ in (74) into the above and rearranging we obtain the following expression for $V_{1}(t)$ :

$$
V_{1}(t)=\frac{\int_{0}^{1} b(x) R(x)\left[\eta_{0}(x, t)+\Phi_{0}(x, t)-\int_{x}^{1} \frac{j_{s, 0}\left(x^{\prime}, t\right)}{\Theta \sigma\left(x^{\prime}\right)} d x^{\prime}\right] d x}{\left(\int_{0}^{1} b(x) R(x) d x\right)}+O(\mathcal{Q}),
$$

and this completes the derivation of the dimensionless corrected multiple particle model, the dimensional version of which is given in (16)-(18) and (20)-(25). 


\section{Conclusions}

We have shown how the widely-used single particle (SP) model can be derived directly from the Doyle-Fuller-Newman (DFN) model, using systematic asymptotic methods as well as how it can be generalised to treat graded electrodes and electrodes with multiple chemistries. In addition, we have shown that while the results of the SP model, and its generalisation the multi-particle (MP) model, give reasonable agreement to the DFN model for small discharge rates the discrepancies become significant at higher rates of discharge. This motivated us to derive a correction term to the SP model, and its generalisation the MP model. We demonstrated that the corrected single particle (cSP) model and its generalisation the corrected multiparticle (cMP) model offer very significantly increased accuracy over the basic SP and MP models, to such a degree that they are able to accurately reproduce discharge curves with C-rates up to around 12C in graphite and 16C in NMC. Perhaps somewhat surprisingly the cSP model also works reasonably well for LFP even though the asymptotic derivation is not applicable to a material with such a flat discharge curve. However the cMP model and the full DFN model are not in such good agreement for a graded LFP electrode with two different particle sizes. Calculating the correction terms in the cSP and cMP models requires that a one-dimensional model for the electrolyte is solved, while still assuming that the particles in a given electrode discharge such that lithium concentrations $c_{s}$ on their surfaces is uniform, across the entire width of the electrode. This means that, while there may be significant variations in the electrolytic lithium concentration $c$ across the width of the cell, the cSP approximation to the DFN model loses its validity if electrolyte depletion occurs (i.e. $c$ approaches zero) in any part of the cell. This is because, in the full DFN model, electrolyte depletion leads to the (de-)intercalation reaction switching off within the depletion region. This is forbidden by the cSP model which requires that the lithium concentration $c_{s}$ on the surface of the electrode particles is uniform across all particles in the electrode.

Throughout the main text of this work we concentrated on a half-cell configuration in order to illustrate for which chemistries, and under which conditions, the cSP model can be expected to work well. We find that for materials such as graphite and NMC, for which the OCV curves are not flat, the approximation is extremely good, but for a material such as LFP, with a flat OCV curve, the approximation is considerably worse, although perhaps better than might be expected from the asymptotic analysis. Motivated by its likely eventual application we have, in Appendix B, stated and derived the model in a full-cell configuration, as well as validating this full-cell model against solutions to the full-cell DFN model.

Both the cSP and cMP models require that, in addition to solving one-dimensional diffusion equations in representative electrode particles, a one-dimensional model for the electrolyte is solved across the cell. It is therefore more complex than the basic SP or MP models but is still much cheaper to solve than the full DFN model. After discretising in space with $M$ mesh points for the electrode particle problem and $N$ for the electrolyte problem it is found that the total number of differential algebraic equations that need to be integrated forward in time for the SP model, cSP model and the DFN model are approximately $M, N+M$ and $N M+N$, respectively. Using a standard black-box integrator, the computational costs scale with the number of equations squared so that for the SP model the cost scales with $M^{2}$, the cSP model the cost scales with $(N+M)^{2}$ and for the DFN model it scales with $(N M+N)^{2}$. Thus it can be seen that though the cSP model is marginally more costly than the SP model 
it is still orders magnitude quicker than the DFN model for any reasonable choice of values of $N$ and $M$. As an example, with $M=50$ and $N=100$, we find that a typical solution of the cSP model, to obtain a full discharge curve in MATLAB using the ode15s DAE solver on a standard desktop computer with 6th generation Intel Core i7 CPU takes approximately $1.5 \mathrm{sec}$. This compares to a computation time of $170 \mathrm{sec}$., for the equivalent calculation, using the full DFN model, and so is two orders of magnitude faster than the DFN solve. Furthermore this disparity becomes even more pronounced with increases in $N$ or $M$. The SP model does not require that the electrolyte equations are solved, and hence it works faster than the cSP model, but the difference in performance is not particularly dramatic. With $M=50$, the SP model takes roughly $0.3 \mathrm{sec}$. of computation time which is approximately 5 times faster than the cSP model for the given parametrisation and computational grid.

So long as the caveats that we have outlined above are respected, the corrected versions of the SP model derived here provide an accurate and fast means of modelling the internal electrochemical process in modern LIB cells. The relative ease with which these simulations can be carried out make this type of model an excellent candidate for use in applications where computational costs need to be kept to a minimum such as battery management systems (e.g. [34]), optimal cell design (e.g. [7]), extension of DFN to 3-macroscopic dimensions (e.g. $[47,10])$, and as a tool in parameter estimation studies (e.g. $[4,41])$.

Acknowledgements GR, IK and JF were supported by the Faraday Institution MultiScale Modelling (MSM) project Grant number EP/S003053/1. MC acknowledges funding from the University Alliance, Doctoral Training Alliance. The authors are very grateful for the help of Dr. Simon O'Kane in fitting data for the electrode and electrolyte properties.

\section{A The Green's function for the single particle problem}

Here we write down the problem for the Green's function $\mathcal{G}(r, x, t ; \tau)$ for the problem (84)(85) that is used to write down the expression for $\left.(\partial / \partial r)\left(D_{s}\left(c_{s, 0}\right) c_{s, 1}\right)\right|_{r=R(x)}$ in (86). We start by seeking a solution $\hat{\mathcal{G}}(r, x, t ; \tau)$ that satisfies the problem

$$
\begin{aligned}
\mathcal{Q} \frac{\partial \hat{\mathcal{G}}}{\partial t}= & \frac{1}{r^{2}} \frac{\partial}{\partial r}\left(r^{2} \frac{\partial}{\partial r}\left(D_{s}\left(c_{s, 0}\right) \hat{\mathcal{G}}\right)\right) \quad \text { in } \quad 0 \leq r \leq R(x), \\
\hat{\mathcal{G}}(r, x, t ; \tau) \quad & \text { bounded on } r=0,\left.\quad \hat{\mathcal{G}}(r, x, t ; \tau)\right|_{r=R(x)}=\delta(t-\tau), \\
\hat{\mathcal{G}}(r, x, t ; \tau)= & 0 \quad \text { for } \quad t \leq \tau .
\end{aligned}
$$

Then by using the superposition principle it is straightforward to show that

$$
c_{s, 1}(r, x, t)=\int_{0}^{\infty} \mathcal{C}_{1}(x, \tau) \hat{\mathcal{G}}(r, x, t ; \tau) d \tau=\int_{0}^{t} \mathcal{C}_{1}(x, \tau) \hat{\mathcal{G}}(r, x, t ; \tau) d \tau
$$


is a solution to the problem (84)-(85). It follows that the quantity for which we wish to obtain an expression: $\left.(\partial / \partial r)\left(D_{s}\left(c_{s, 0}\right) c_{s, 1}\right)\right|_{r=R(x)}$ can be written in the form

$$
\begin{array}{r}
\left.\frac{\partial}{\partial r}\left(D_{s}\left(c_{s, 0}\right) c_{s, 1}\right)\right|_{r=R(x)}=\int_{0}^{t} \mathcal{C}_{1}(x, \tau) \mathcal{G}(x, t ; \tau) d \tau \\
\text { where } \quad \mathcal{G}(x, t ; \tau)=\left.\left[D_{s}^{\prime}\left(c_{s, 0}\right) \frac{\partial c_{s, 0}}{\partial r} \hat{\mathcal{G}}(r, x, t ; \tau)+D_{s}\left(c_{s, 0}\right) \frac{\partial \hat{\mathcal{G}}}{\partial r}(r, x, t ; \tau)\right]\right|_{r=R(x)} .
\end{array}
$$

\section{B Full Cell discharge models}

In this section the full cell DFN model is formulated and the analogous cSP (and cMP) model written down. In order to verify the validity the numerical method used here comparison is made between solution of the full cell DFN model and experimental discharge curve from [15]. Similarly the validity of the full cell cSP model is then confirmed by the comparison between our solutions of the full cell DFN model and solution of the full cell cSP model. Here we consider a full cell in which:

$$
\begin{array}{ll}
\text { The anode occupies } & L_{1}<x<L_{2}, \\
\text { the separator occupies } & L_{2}<x<L_{3}, \\
\text { the cathode occupies } & L_{3}<x<L_{4} .
\end{array}
$$

The cell was parametrised according to the measurements carried out by Ecker et al. [14] on their experimental cell. In their work, the authors considered a commercial pouch cell produced by Kokam. Their pouch cell has a nominal capacity of 7.5Ah and operating voltages between $2.7 \mathrm{~V}$ and $4.2 \mathrm{~V}$. According to the manufacturer, the cell consists of an $\mathrm{LiC}_{6}$ anode, an $\mathrm{Li}(\mathrm{NiMnCo}) \mathrm{O}_{2}$ cathode and an $\mathrm{LiPF}_{6}$ electrolyte. However, spectroscopy measurements performed by the authors, revealed an absence of any manganese in the cathode material. These data showed that the cathode comprises $60 \%$ cobalt and $40 \%$ nickel, and therefore, following Ecker et al. 's notation we denote it as $\mathrm{Li}\left(\mathrm{Ni}_{0.4} \mathrm{Co}_{0.6}\right) \mathrm{O}_{2}$. Experimental diffusivity and conductivity measurements of the electrolyte properties and the nonlinear lithium diffusivities in the electrodes, with the corresponding theoretical fits to this data, are given in the figure 2. The equilibrium potentials of the graphite anode and the $\mathrm{Li}\left(\mathrm{Ni}_{0.4} \mathrm{Co}_{0.6}\right) \mathrm{O}_{2}$ cathode can be found in figure 3, and the remaining electrode and electrolyte parameters are summarised in table 1.

\section{The full cell DFN model}

The full cell DFN model (analogous to the half cell model (1)-(10)) is given below. 
Macroscopic equations. These are as follows:

$$
\begin{gathered}
\epsilon_{l}(x) \frac{\partial c}{\partial t}+\frac{\partial N_{-}}{\partial x}=0, \quad N_{-}=-\mathcal{B}(x) D(c) \frac{\partial c}{\partial x}-\left(1-t^{+}\right) \frac{j}{F} \quad \text { in } \quad L_{1}<x<L_{4} . \\
\frac{\partial j}{\partial x}=b(x) j_{n}, \quad j=-\mathcal{B}(x) \kappa(c)\left(\frac{\partial \Phi}{\partial x}-\frac{2 R_{g} T}{F} \frac{1-t^{+}}{c} \frac{\partial c}{\partial x}\right) \quad \text { in } \quad L_{1}<x<L_{4}, \\
\frac{\partial j_{a}}{\partial x}=-b(x) j_{n}, \quad j_{a}=-\sigma \frac{\partial \Phi_{a}}{\partial x} \quad \text { in } \quad L_{1}<x<L_{2}, \\
\frac{\partial j_{c}}{\partial x}=-b(x) j_{n}, \quad j_{c}=-\sigma \frac{\partial \Phi_{c}}{\partial x} \quad \text { in } \quad L_{3}<x<L_{4}, \\
j_{n}=\left\{\begin{array}{r}
2 F k c^{1 / 2}\left(\left.c_{a}\right|_{r=R_{a}(x)}\right)^{1 / 2}\left(c_{a}^{\max }-\left.c_{a}\right|_{r=R_{a}(x)}\right)^{1 / 2} \sinh \left(\frac{F \eta_{a}}{2 R_{g} T}\right) \text { in } \quad L_{1} \leq x<L_{2}, \\
2 F k c^{1 / 2}\left(\left.c_{c}\right|_{r=R_{c}(x)}\right)^{1 / 2}\left(c_{c}^{\max }-\left.c_{c}\right|_{r=R_{c}(x)}\right)^{1 / 2} \sinh \left(\frac{F \eta_{c}}{2 R_{g} T}\right) \quad \text { in } \quad L_{2}<x<L_{3}, \\
\eta_{a}=\Phi_{a}-\Phi-U_{e q, a}\left(\left.c_{a}\right|_{r=R_{a}(x)}\right), \quad \eta_{c}=\Phi_{c}-\Phi-U_{e q, c}\left(\left.c_{c}\right|_{r=R_{c}(x)}\right) .
\end{array}\right.
\end{gathered}
$$

Here the notation is as before except that, in order to distinguish between electrode variables in the anode and electrode variables in the cathode, we replace the variables $\Phi_{s}, j_{s}, c_{s}$ by $\Phi_{a}, j_{a}, c_{a}$ in the anode $\left(L_{1}<x<L_{2}\right)$ and by $\Phi_{c}, j_{c}, c_{c}$ in the cathode $\left(L_{3}<x<\right.$ $\left.L_{4}\right)$. Furthermore the electrode particles in the anode and cathode have different electrical properties and so are characterised by different equilibrium potential functions, $U_{e q, a}\left(c_{a}\right)$ in the anode and $U_{e q, c}\left(c_{c}\right)$ in the cathode.

Macroscopic boundary and interface conditions Here the macroscopic boundary and interface conditions on the model

$$
\begin{aligned}
& \left.j_{a}\right|_{x=L_{1}}=\frac{I(t)}{A},\left.\quad N_{-}\right|_{x=L_{1}}=0,\left.\quad j\right|_{x=L_{1}}=0, \\
& \left.j_{a}\right|_{x=L_{2}}=0 \\
& \left.j_{c}\right|_{x=L_{3}}=0, \\
& \left.j_{c}\right|_{x=L_{4}}=\frac{I(t)}{A},\left.\quad N_{-}\right|_{x=L_{4}}=0,\left.\quad j\right|_{x=L_{4}}=0 .
\end{aligned}
$$

representing galvanostatic discharge at a current $I(t)$ which flows into the anode current collector on $x=L_{1}$ through the anode particles and out through the cathode current collector on $x=L_{4}$ through the cathode particles.

Microscopic equations and boundary conditions The microscopic equations and boundary conditions on the model are analogous to those on the half cell model being given 
by

$$
\begin{aligned}
& \left.\begin{array}{l}
\frac{\partial c_{a}}{\partial t}=\frac{1}{r^{2}} \frac{\partial}{\partial r}\left(r^{2} D_{a}\left(c_{a}\right) \frac{\partial c_{a}}{\partial r}\right) \quad \text { in } 0<r<R_{a}(x) \\
c_{a} \text { bounded on } r=0, \quad-\left.D_{a}\left(c_{a}\right) \frac{\partial c_{a}}{\partial r}\right|_{r=R_{a}(x)}=\frac{j_{n}}{F}
\end{array}\right\} \text { in } L_{1}<x<L_{2}, \\
& \left.\begin{array}{l}
\frac{\partial c_{c}}{\partial t}=\frac{1}{r^{2}} \frac{\partial}{\partial r}\left(r^{2} D_{c}\left(c_{c}\right) \frac{\partial c_{c}}{\partial r}\right) \quad \text { in } 0<r<R_{c}(x) \\
c_{c} \text { bounded on } r=0, \quad-\left.D_{c}\left(c_{c}\right) \frac{\partial c_{c}}{\partial r}\right|_{r=R_{c}(x)}=\frac{j_{n}}{F}
\end{array}\right\} \text { in } L_{3}<x<L_{4},
\end{aligned}
$$

where $R_{a}$ and $R_{c}$ are the radii of the anode and cathode particles respectively.

The full cell potential The results of solution to the full cell DFN model (with appropriate initial conditions) and a specified galvanostatic current $I(t)$ can be used to compute the potentials of the anode and cathode current collectors $V_{a}$ and $V_{c}$, respectively via the relations

$$
V_{a}(t)=\left.\Phi_{a}\right|_{x=L_{1}}, \quad V_{c}(t)=\left.\Phi_{c}\right|_{x=L_{4}} .
$$

and hence the potential drop across the full cell is given by

$$
V(t)=V_{c}(t)-V_{a}(t)
$$

\section{The full cell cSP and cMP models}

The full cell cSP and cMP models can be derived analogously to the half cell cSP and cMP models in $\S 5$. The results of this derivation can be summarised as follows. The potential difference $V(t)$ across the cell is given by

$$
\begin{aligned}
V(t) & =V_{c}(t)-V_{a}(t), \quad \text { where } \\
V_{a} & =U_{e q, a}\left(\mathcal{C}_{a}(t)\right)+\frac{1}{\int_{L_{1}}^{L_{2}} b(x) R_{a}(x) d x}\left(\int_{L_{1}}^{L_{2}} b(x) R_{a}(x)\left[\eta_{a}+\Phi+\int_{L_{1}}^{x} \frac{j_{a}}{\sigma_{a}} d x\right] d x\right), \\
V_{c} & =U_{e q, c}\left(\mathcal{C}_{c}(t)\right)+\frac{1}{\int_{L_{3}}^{L_{4}} b(x) R_{c}(x) d x}\left(\int_{L_{3}}^{L_{4}} b(x) R_{c}(x)\left[\eta_{c}+\Phi-\int_{x}^{L_{4}} \frac{j_{c}}{\sigma_{c}} d x\right] d x\right),
\end{aligned}
$$

Here the unspecified functions $\mathcal{C}_{a}(t), \mathcal{C}_{c}(t), \eta_{a}(x, t), \eta_{c}(x, t), j_{a}(x, t), j_{c}(x, t)$ and $\Phi(x, t)$ appearing in this model are determined from the solutions of the two microscopic electrode particle problems

$$
\begin{gathered}
\frac{\partial c_{a}}{\partial t}=\frac{1}{r^{2}} \frac{\partial}{\partial r}\left(r^{2} D_{a}\left(c_{a}\right) \frac{\partial c_{a}}{\partial r}\right) \quad \text { in } \quad 0 \leq r \leq R_{a}(x), \\
c_{a} \text { bounded on } r=0,\left.\quad c_{a}\right|_{r=R_{a}}=\mathcal{C}_{a}(t),\left.\quad c_{a}\right|_{t=0}=c_{a, \text { init }}, \\
\int_{L_{1}}^{L_{2}} b(x) j_{n}(x, t) d x=\frac{I(t)}{A} \quad \text { where } \quad j_{n}=-\left.F D_{a}\left(c_{a}\right) \frac{\partial c_{a}}{\partial r}\right|_{r=R_{a}(x)}
\end{gathered}
$$


and

$$
\begin{gathered}
\frac{\partial c_{c}}{\partial t}=\frac{1}{r^{2}} \frac{\partial}{\partial r}\left(r^{2} D_{c}\left(c_{c}\right) \frac{\partial c_{c}}{\partial r}\right) \quad \text { in } \quad 0 \leq r \leq R_{c}(x), \\
c_{c} \text { bounded on } r=0,\left.\quad c_{c}\right|_{r=R_{c}}=\mathcal{C}_{c}(t),\left.\quad c_{c}\right|_{t=0}=c_{a, \text { init }}, \\
\int_{L_{3}}^{L_{4}} b(x) j_{n}(x, t) d x=-\frac{I(t)}{A} \quad \text { where } \quad j_{n}=-\left.F D_{c}\left(c_{c}\right) \frac{\partial c_{c}}{\partial r}\right|_{r=R_{c}(x)}
\end{gathered}
$$

and the macroscopic electrolyte problem in $L_{1}<x<L_{4}$

$$
\begin{array}{r}
\epsilon_{l}(x) \frac{\partial c}{\partial t}+\frac{\partial N_{-}}{\partial x}=0, \quad N_{-}=-\mathcal{B}(x) D(c) \frac{\partial c}{\partial x}-\left(1-t^{+}\right) \frac{j}{F} \\
\frac{\partial j}{\partial x}=b(x) j_{n}(x, t), \quad j=-\mathcal{B}(x) \kappa(c)\left(\frac{\partial \Phi}{\partial x}-\frac{2 R_{g} T}{F} \frac{1-t^{+}}{c} \frac{\partial c}{\partial x}\right) \\
\left.j\right|_{x=L_{1}}=\frac{I(t)}{A},\left.\quad N_{-}\right|_{x=L_{1}}=0,\left.\quad N_{-}\right|_{x=L_{4}}=0,\left.\quad j\right|_{x=L_{4}}=\frac{I(t)}{A} \\
\text { and }\left.\quad c\right|_{t=0}=c_{\text {init }} .
\end{array}
$$

In particular the electrode currents $j_{a}$ and $j_{c}$, and the overpotentials $\eta_{a}$ and $\eta_{c}$ are given in terms of the solutions to these problems by

$$
\begin{aligned}
& \left.\begin{array}{l}
j_{a}(x, t)=\frac{I}{A}-j(x, t) \\
\eta_{a}(x, t)=2 \frac{R_{g} T}{F} \operatorname{arcsinh}\left(\frac{j_{n}(x, t)}{2 F k\left[\left(c_{a}^{\max }-\mathcal{C}_{a}(t)\right) \mathcal{C}_{a}(t) c\right]^{1 / 2}}\right)
\end{array}\right\} \text { in } L_{1}<x<L_{2}, \\
& j_{c}(x, t)=\frac{I}{A}-j(x, t) \\
& \left.\eta_{c}(x, t)=2 \frac{R_{g} T}{F} \operatorname{arcsinh}\left(\frac{j_{n}(x, t)}{2 F k\left[\left(c_{c}^{\max }-\mathcal{C}_{c}(t)\right) \mathcal{C}_{c}(t) c\right]^{1 / 2}}\right)\right\} \quad \text { in } L_{3}<x<L_{4}
\end{aligned}
$$

Results In order to compare the full cell DFN, SP and cSP models to experimental data the models were parametrised according to experiments described in Ecker et al. [15] for a commercial 7.5 Ah cell produced by Kokam. Simulated and measured discharge curves at room temperature and different discharge rates are shown in figure 8.

It can be noted that the simulated curves capture the experimental profile very well. For $1 \mathrm{C}$ not only is the total capacity in good agreement with the measurements but the standard DFN, SP and cSP models all reproduce the shape of the experimental discharge curve. For higher discharge rates $(5 \mathrm{C})$ the discrepancy between SP and the DFN models is much higher but the full cell cSP model still agrees very well with the DFN discharge profile and is also very close to the experimental data.

In addition, in figure 9, the results of the full cell DFN, SP and cSP models are compared to those of the generalised SPMe model formulated in [26], which is based on a different asymptotic approach to the one used here. Notably the cSP model (from this paper) agrees extremely well with the full cell DFN results for all discharge rates between $1 \mathrm{C}$ and $7.5 \mathrm{C}$ 

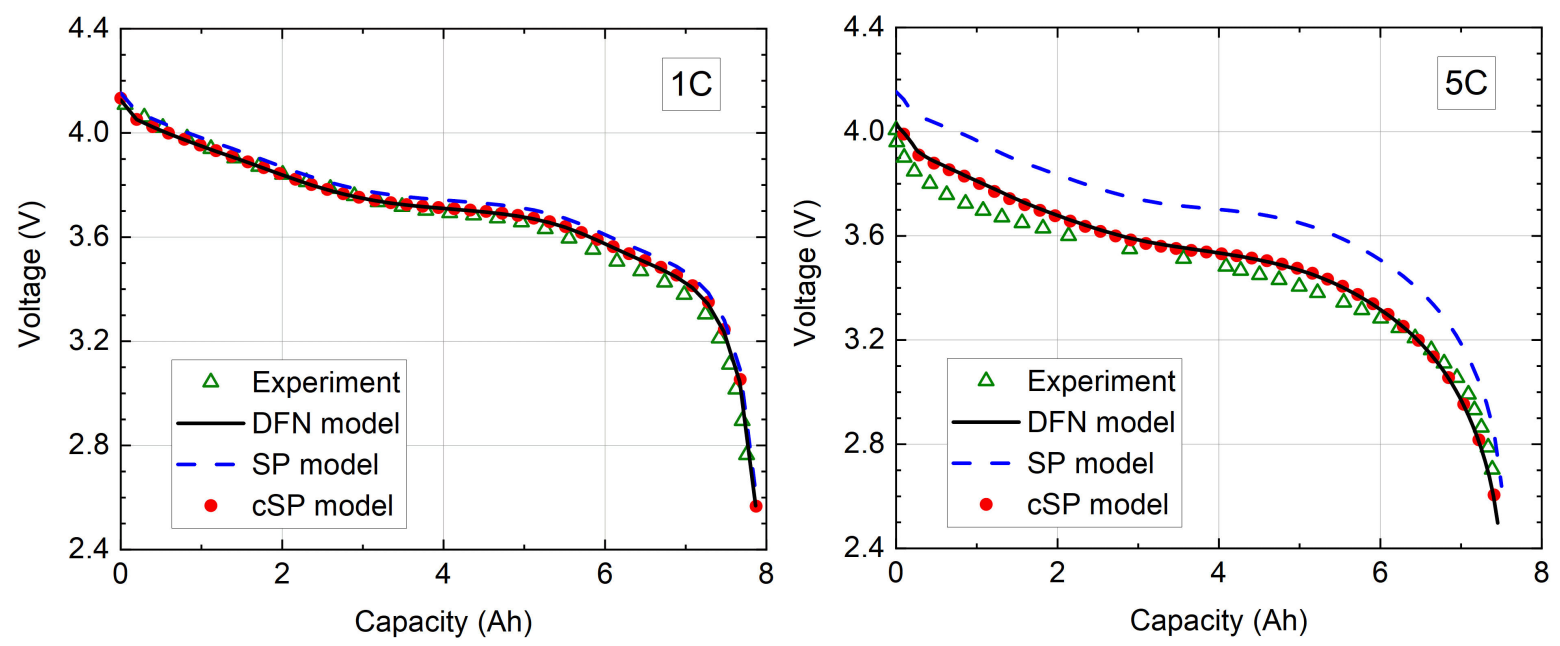

Figure 8: Comparison between solution of the full cell DFN model, corresponding SP and cSP models and experimental discharge curve from Ecker et al. [15] at 1C (left) and 5C (right) discharge rates.

whereas there are significant differences between the SPMe results (developed in [26]) and the DFN results at higher discharge rates, see figure 9. This, we believe, is due to a number of ad-hoc assumptions made in [26] in order to derive their generalised SPMe model, given in equation (48) of that work. For instance, unlike the cSP model, in the SPMe model the electrolyte is described by a linear diffusion equation with some constant sources (for constant current). For rates higher than about 7.5C the electrolyte begins to deplete in the cathode (lithium concentration becomes very close to zero) and for this reason the agreement between the cSP model and the DFN model becomes worse at these higher discharge rates. In terms of compute time the cSP model takes slightly longer, at around $1.5 \mathrm{~s}$, than the SPMe model, at around $0.8 \mathrm{~s}$, when solved using the same grid size and solver. However the much greater accuracy of cSPM over SPMe is certainly worth this slight increase in computational cost.

\section{References}

[1] P. Bai, D. A. Cogswell, and M. Z. Bazant, Suppression of phase separation in $\mathrm{LiFePO}_{4}$ nanoparticles during battery discharge, Nano Letters, 11 (2011), pp. 48904896.

[2] P. Biesheuvelnd and M. Z. Bazant, Nonlinear dynamics of capacitive charging and desalination by porous electrodes, Physical Review E, 81(3):031502 (2010).

[3] P. Biesheuvel, Y. Fu and M. Z. Bazant, Diffuse charge and faradaic reactions in porous electrodes, Physical Review E, 83(6):061507 (2011).

[4] A. M. Bizeray, J.-H. Kim, S. R. Duncan, and D. A. Howey, Identifiability and parameter estimation of the single particle lithium-ion battery model, IEEE Transactions on Control Systems Technology, (2018), pp. 1-16. 

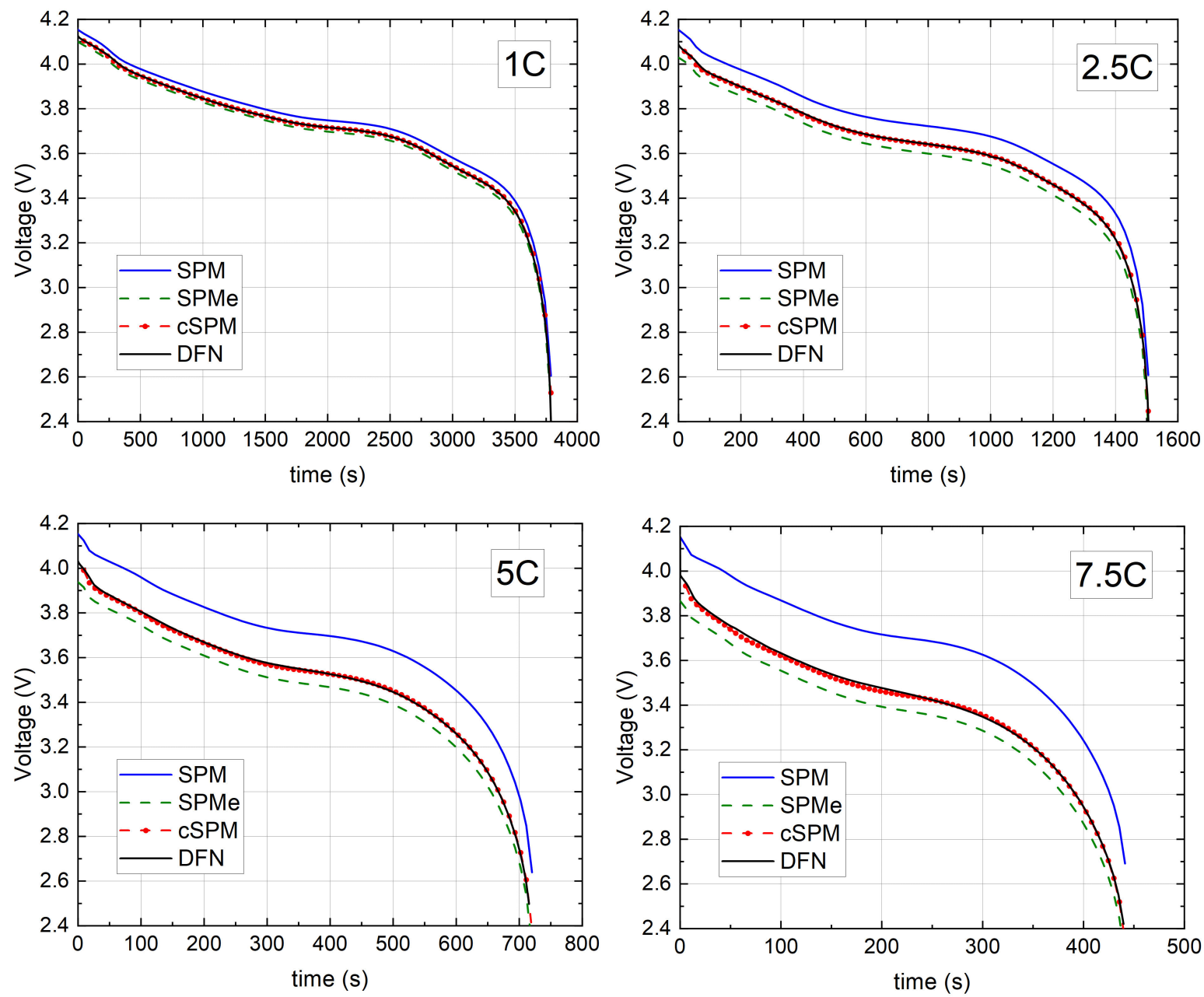

Figure 9: Comparison between solution of the full cell DFN model, corresponding SP, cSP models and SPMe [26] at different discharge rates.

[5] G. E. Blomgren, The development and future of lithium ion batteries, Journal of The Electrochemical Society, 164 (2017), pp. A5019-A5025.

[6] B. D. Bruggeman, Calculation of different physical constants of heterogeneous substances. i. dielectric constants and conductivities of mixed bodies of isotropic substances, Annalen der Physik, 416 (1935), pp. 636-664.

[7] C. Cheng, R. Drummond, S. R. Duncan, and P. S. Grant, Micro-scale graded electrodes for improved dynamic and cycling performance of Li-ion batteries, Journal of Power Sources, 413 (2019), pp. 59-67.

[8] F. Ciucci and W. LaI, Derivation of micro/macro lithium battery models from homogenization, Transport in Porous Media, 88 (2011), pp. 249-270.

[9] D. A. Cogswell and M. Z. Bazant, Theory of coherent nucleation in phaseseparating nanoparticles, Nano Letters, 13 (2013), pp. 3036-3041. 
[10] T. Danner, M. Singh, S. Hein, J. Kaiser, H. Hahn, and A. Latz, Thick electrodes for Li-ion batteries: A model based analysis, Journal of Power Sources, 334 (2016), pp. 191-201.

[11] S. Dargaville and T. Farrell, A comparison of mathematical models for phasechange in high-rate $\mathrm{LiFePO}_{4}$ cathodes, Electrochimica Acta, 111 (2013), pp. 474-490.

[12] M. Doyle, T. F. Fuller, And J. Newman, Modeling of galvanostatic charge and discharge of the lithium/polymer/insertion cell, Journal of the Electrochemical Society, 140 (1993), pp. 1526-1533.

[13] M. Doyle, J. Newman, A. S. Gozdz, C. N. Schmutz, and J.-M. Tarascon, Comparison of modeling predictions with experimental data from plastic lithium ion cells, Journal of the Electrochemical Society, 143 (1996), pp. 1890-1903.

[14] M. Ecker, T. K. D. Tran, P. Dechent, S. Käbitz, A. Warnecke, and D. U. SAUER, Parameterization of a physico-chemical model of a lithium-ion battery i. determination of parameters, Journal of The Electrochemical Society, 162 (2015), pp. A1836A1848.

[15] M. Ecker, S. KäBitz, I. Laresgoiti, and D. U. Sauer, Parameterization of a physico-chemical model of a lithium-ion battery ii. model validation, Journal of The Electrochemical Society, 162 (2015), pp. A1849-A1857.

[16] T. R. Ferguson and M. Z. Bazant, Phase transformation dynamics in porous battery electrodes, Electrochimica Acta, 146 (2014), pp. 89-97.

[17] J. Foster, A. Gully, H. Liu, S. Krachkovskiy, Y. Wu, S. Schougaard, M. Jiang, G. Goward, G. Botton, and B. Protas, Homogenization study of the effects of cycling on the electronic conductivity of commercial lithium-ion battery cathodes, The Journal of Physical Chemistry C, 119 (2015), pp. 12199-12208.

[18] A. A. Franco, Multiscale modelling and numerical simulation of rechargeable lithium ion batteries: concepts, methods and challenges, RSC Advances, 3 (2013), pp. 1302713058.

[19] T. F. Fuller, M. Doyle, And J. Newman, Simulation and optimization of the dual lithium ion insertion cell, Journal of the Electrochemical Society, 141 (1994), pp. 1-10.

[20] Y. Guo, R. B. Smith, Z. Yu, D. K. Efetov, J. Wang, P. Kim, M. Z. Bazant AND L. E. BRUS, Li intercalation into graphite: direct optical imaging and CahnHilliard reaction dynamics, The Journal of Physical Chemistry Letters, 7:11 (2106), pp. 2151-2156.

[21] M. G. Hennessy AND I. R. Moyles, Asymptotic reduction, solution, and homogenisation of a thermo-electrochemical model for a lithium-ion battery, Applied Mathematical Modelling, 80 (2020), pp. 724-754. 
[22] A. Jokar, B. Rajabloo, M. Désilets, and M. Lacroix, Review of simplified pseudo-two-dimensional models of lithium-ion batteries, Journal of Power Sources, 327 (2016), pp. 44-55.

[23] S. A. Krachkovskiy, J. M. Foster, J. D. Bazak, B. J. Balcom, and G. R. GOWARD, Operando mapping of Li concentration profiles and phase transformations in graphite electrodes by magnetic resonance imaging and nuclear magnetic resonance spectroscopy, Journal of Physical Chemistry C, 122:38 (2018), pp. 21784-21791.

[24] S. Kosch, Y. Zhao, J. Sturm, J. Schuster, G. Mulder, E. Ayerbe, And A. Jossen, A computationally efficient multi-scale model for Lithium-ion cells, Journal of The Electrochemical Society, 165 (2018), pp. A2374-A2388.

[25] Y. Li, F. El Gabaly, T. R. Ferguson, R. B. Smith, N. C. Bartelt, J. D. Sugar, K. R. Fenton, D. A. Cogswell, A. D. Kilcoyne, T. Tyliszczak, ET AL., Current-induced transition from particle-by-particle to concurrent intercalation in phase-separating battery electrodes, Nature Materials, 13 (2014), p. 1149.

[26] S. G. Marquis, V. Sulzer, R. Timms, C. P. Please, and S. J. Chapman, An asymptotic derivation of a single particle model with electrolyte, arXiv preprint arXiv:1905.12553, (2019).

[27] S. J. Moura, F. B. Argomedo, R. Klein, A. Mirtabatabaei, and M. Krstic, Battery state estimation for a single particle model with electrolyte dynamics, IEEE Transactions on Control Systems Technology, 25 (2016), pp. 453-468.

[28] I. R. Moyles, M. G. Hennessy, T. G. Myers, and B. R. Wetton, Asymptotic reduction of a porous electrode model for lithium-ion batteries, SIAM Journal on Applied Mathematics, 79(4) (2019), pp. 1528-1549.

[29] R. Mukherjee, R. Krishnan, T. Lu, and N. Koratkar, Nanostructured electrodes for high-power lithium ion batteries, Nano Energy, 1:4 (2012), pp. 518-533.

[30] J. Newman and K.E. Thomas-Alyea, Electrochemical Systems, vol. 1, Prentice Hall, New Jersey, 2004.

[31] J. Newman and W. Tiedemann, Porous-electrode theory with battery applications, AIChE Journal, 21 (1975), pp. 25-41.

[32] — Potential and current distribution in electrochemical cells interpretation of the half-cell voltage measurements as a function of reference-electrode location, Journal of The Electrochemical Society, 140 (1993), pp. 1961-1968.

[33] K. Persson, V. A. Sethuraman, L. J. Hardwick, Y. Hinuma, Y. S. Meng, A. Van Der Ven, V. Srinivasan, R. Kostecki, and G. Ceder, Lithium diffusion in graphitic carbon, The Journal of Physical Chemistry Letters, 1 (2010), pp. 1176-1180.

[34] G. L. PLETT, High-performance battery-pack power estimation using a dynamic cell model, IEEE Transactions on Vehicular Technology, 53 (2004), pp. 1586-1593. 
[35] S. K. Rahimian, S. Rayman, and R. E. White, Extension of physics-based single particle model for higher charge-discharge rates, Journal of Power Sources, 224 (2013), pp. 180-194.

[36] R. RANOm, Mathematical modelling of Lithium ion batteries, PhD thesis, University of Southampton, 2014.

[37] G. Richardson, G. Denuault, and C. Please, Multiscale modelling and analysis of lithium-ion battery charge and discharge, Journal of Engineering Mathematics, 72 (2012), pp. 41-72.

[38] G. Richardson, I. Korotkin, R. Ranom. M. Castle, J. M. Foster, Generalised single particle models for high-rate operation of graded lithium-ion electrodes: systematic derivation and validation, arXiv:1907.09410, 2019.

[39] G. Richardson, J. M. Foster, R. Ranom, C. P. Please and A. M. Ramos, Charge transport modelling of lithium ion batteries, arxiv, 2019.

[40] M. SchmUCK, Upscaling of solid-electrolyte composite intercalation cathodes for energy storage systems, Applied Mathematics Research eXpress, 2017 (2017), pp. 402-430.

[41] A. K. Sethurajan, J. M. Foster, G. Richardson, S. A. Krachkovskiy, J. D. BAZAK, G. R. GOWARD, AND B. PROTAS, Incorporating dendrite growth into continuum models of electrolytes: Insights from nmr measurements and inverse modeling, Journal of The Electrochemical Society, 166 (2019), pp. A1591-A1602.

[42] V. Srinivasan and J. Newman, Design and Optimization of a Natural Graphite/Iron Phosphate Lithium-Ion Cell, Journal of The Electrochemical Society, 151 (2004), pp. A1530-A1538.

[43] K. E. Thomas, J. Newman, and R. M. Darling, Mathematical modeling of lithium batteries, in Advances in lithium-ion batteries, Springer, 2002, pp. 345-392.

[44] J. Vetter, P. Novák, M. R. Wagner, C. Veit, K.-C. Möller, J. Besenhard, M. Winter, M. Wohlfahrt-Mehrens, C. Vogler, and A. Hammouche, Ageing mechanisms in lithium-ion batteries, Journal of Power Sources, 147 (2005), pp. 269-281.

[45] Q. Wang, P. Ping, X. Zhao, G. Chu, J. Sun, and C. Chen, Thermal runaway caused fire and explosion of lithium ion battery, Journal of Power Sources, 208 (2012), pp. $210-224$.

[46] Q. Wu, W. Lu, AND J. Prakash, Characterization of a commercial size cylindrical Li-ion cell with a reference electrode, Journal of Power Sources, 88 (2000), pp. 237-242.

[47] J. Yi, U. S. Kim, C. B. Shin, T. Han, And S. PARK, Three-dimensional thermal modeling of a lithium-ion battery considering the combined effects of the electrical and thermal contact resistances between current collecting tab and lead wire, Journal of the Electrochemical Society, 160 (2013), pp. A437-A443. 
[48] F. YU KANG, J. MA, AND B. HUA LI, Effects of carbonaceous materials on the physical and electrochemical performance of a $\mathrm{LiFePO}_{4}$ cathode for lithium-ion batteries, New Carbon Materials, 26 (2011), pp. 161-170.

[49] W. Zhao, J. Yi, P. He And H. Zhou, Solid-state electrolytes for lithium-ion batteries: Fundamentals, challenges and perspectives., Electrochemical Energy Reviews, 1-32 (2019).

[50] G. Zubi, R. Dufo-Lopez, M. Carvalho, and G. Pasaoglu, The lithium-ion battery: State of the art and future perspectives, Renewable and Sustainable Energy Reviews, 89 (2018), pp. 292-308. 